\title{
Nonautonomous Solitons: Applications from Nonlinear Optics to BEC and Hydrodynamics
}

\author{
T. L. Belyaeva ${ }^{1}$ and V. N. Serkin ${ }^{2}$ \\ ${ }^{1}$ Universidad Autónoma del Estado de México \\ ${ }^{2}$ Benemerita Universidad Autónoma de Puebla \\ Mexico
}

\section{Introduction}

Nonlinear science is believed by many outstanding scientists to be the most deeply important frontier for understanding Nature (Christiansen et al., 2000; Krumhansl, 1991). The interpenetration of main ideas and methods being used in different fields of science and technology has become today one of the decisive factors in the progress of science as a whole. Among the most spectacular examples of such an interchange of ideas and theoretical methods for analysis of various physical phenomena is the problem of solitary wave formation in nonautonomous and inhomogeneous dispersive and nonlinear systems. These models are used in a variety of fields of modern nonlinear science from hydrodynamics and plasma physics to nonlinear optics and matter waves in Bose-Einstein condensates.

The purpose of this Chapter is to show the progress that is being made in the field of the exactly integrable nonautonomous and inhomogeneous nonlinear evolution equations possessing the exact soliton solutions. These kinds of solitons in nonlinear nonautonomous systems are well known today as nonautonomous solitons. Most of the problems considered in the present Chapter are motivated by their practical significance, especially the hydrodynamics applications and studies of possible scenarios of generations and controlling of monster (rogue) waves by the action of different nonautonomous and inhomogeneous external conditions.

Zabusky and Kruskal (Zabusky \& Kruskal, 1965) introduced for the first time the soliton concept to characterize nonlinear solitary waves that do not disperse and preserve their identity during propagation and after a collision. The Greek ending "on" is generally used to describe elementary particles and this word was introduced to emphasize the most remarkable feature of these solitary waves. This means that the energy can propagate in the localized form and that the solitary waves emerge from the interaction completely preserved in form and speed with only a phase shift. Because of these defining features, the classical soliton is being considered as the ideal natural data bit. It should be emphasized that today, the optical soliton in fibers presents a beautiful example in which an abstract mathematical concept has produced a large impact on the real world of high technologies (Agrawal, 2001; Akhmediev, 1997; 2008; Dianov et al., 1989; Hasegawa, 1995; 2003; Taylor, 1992).

Solitons arise in any physical system possessing both nonlinearity and dispersion, diffraction or diffusion (in time or/and space). The classical soliton concept was developed for nonlinear and dispersive systems that have been autonomous; namely, time has only played the role of 
the independent variable and has not appeared explicitly in the nonlinear evolution equation. A not uncommon situation is one in which a system is subjected to some form of external time-dependent force. Such situations could include repeated stress testing of a soliton in nonuniform media with time-dependent density gradients.

Historically, the study of soliton propagation through density gradients began with the pioneering work of Tappert and Zabusky (Tappert \& Zabusky, 1971). As early as in 1976 Chen and Liu (Chen, 1976; 1978) substantially extended the concept of classical solitons to the accelerated motion of a soliton in a linearly inhomogeneous plasma. It was discovered that for the nonlinear Schrödinger equation model (NLSE) with a linear external potential, the inverse scattering transform (IST) method can be generalized by allowing the time-varying eigenvalue (TVE), and as a consequence of this, the solitons with time-varying velocities (but with time invariant amplitudes) have been predicted (Chen, 1976; 1978). At the same time Calogero and Degaspieris (Calogero, 1976; 1982) introduced a general class of soliton solutions for the nonautonomous Korteweg-de Vries $(\mathrm{KdV})$ models with varying nonlinearity and dispersion. It was shown that the basic property of solitons, to interact elastically, was also preserved, but the novel phenomenon was demonstrated, namely the fact that each soliton generally moves with variable speed as a particle acted by an external force rather than as a free particle (Calogero, 1976; 1982). In particular, to appreciate the significance of this analogy, Calogero and Degaspieris introduced the terms boomeron and trappon instead of classical $\mathrm{KdV}$ solitons (Calogero, 1976; 1982). Some analytical approaches for the soliton solutions of the NLSE in the nonuniform medium were developed by Gupta and Ray (Gupta, 1981), Herrera (Herrera, 1984), and Balakrishnan (Balakrishnan, 1985). More recently, different aspects of soliton dynamics described by the nonautonomous NLSE models were investigated in (Serkin \& Hasegawa, 2000a;b; 2002; Serkin et al., 2004; 2007; 2001a;b). In these works, the "ideal" soliton-like interaction scenarios among solitons have been studied within the generalized nonautonomous NLSE models with varying dispersion, nonlinearity and dissipation or gain. One important step was performed recently by Serkin, Hasegawa and Belyaeva in the Lax pair construction for the nonautonomous nonlinear Schrödinger equation models (Serkin et al., 2007). Exact soliton solutions for the nonautonomous NLSE models with linear and harmonic oscillator potentials substantially extend the concept of classical solitons and generalize it to the plethora of nonautonomous solitons that interact elastically and generally move with varying amplitudes, speeds and spectra adapted both to the external potentials and to the dispersion and nonlinearity variations. In particular, solitons in nonautonomous physical systems exist only under certain conditions and varying in time nonlinearity and dispersion cannot be chosen independently; they satisfy the exact integrability conditions. The law of soliton adaptation to an external potential has come as a surprise and this law is being today the object of much concentrated attention in the field. The interested reader can find many important results and citations, for example, in the papers published recently by Zhao et al. (He et al., 2009; Luo et al., 2009; Zhao et al., 2009; 2008), Shin (Shin, 2008) and (Kharif et al., 2009; Porsezian et al., 2007; Yan, 2010).

How can we determine whether a given nonlinear evolution equation is integrable or not? The ingenious method to answer this question was discovered by Gardner, Green, Kruskal and Miura (GGKM) (Gardner et al., 1967). Following this work, Lax (Lax, 1968) formulated a general principle for associating of nonlinear evolution equations with linear operators, so that the eigenvalues of the linear operator are integrals of the nonlinear equation. Lax developed the method of inverse scattering transform (IST) based on an abstract formulation of evolution equations and certain properties of operators in a Hilbert space, some of which 
are well known in the context of quantum mechanics. Ablowitz, Kaup, Newell, Segur (AKNS) (Ablowitz et al., 1973) have found that many physically meaningful nonlinear models can be solved by the IST method.

In the traditional scheme of the IST method, the spectral parameter $\Lambda$ of the auxiliary linear problem is assumed to be a time independent constant $\Lambda_{t}^{\prime}=0$, and this fact plays a fundamental role in the development of analytical theory (Zakharov, 1980). The nonlinear evolution equations that arise in the approach of variable spectral parameter, $\Lambda_{t}^{\prime} \neq 0$, contain, as a rule, some coefficients explicitly dependent on time. The IST method with variable spectral parameter makes it possible to construct not only the well-known models for nonlinear autonomous physical systems, but also discover many novel integrable and physically significant nonlinear nonautonomous equations.

In this work, we clarify our algorithm based on the Lax pair generalization and reveal generic properties of nonautonomous solitons. We consider the generalized nonautonomous NLSE and $\mathrm{KdV}$ models with varying dispersion and nonlinearity from the point of view of their exact integrability. It should be stressed that to test the validity of our predictions, the experimental arrangement should be inspected to be as close as possible to the optimal map of parameters, at which the problem proves to be exactly integrable (Serkin \& Hasegawa, 2000a;b; 2002). Notice, that when Serkin and Hasegawa formulated their concept of solitons in nonautonomous systems (Serkin \& Hasegawa, 2000a;b; 2002), known today as nonautonomous solitons and SH-theorems (Serkin \& Hasegawa, 2000a;b; 2002) published for the first time in (Serkin \& Hasegawa, 2000a;b; 2002), they emphasized that "the methodology developed provides for a systematic way to find an infinite number of the novel stable bright and dark "soliton islands" in a "sea of solitary waves" with varying dispersion, nonlinearity, and gain or absorption" (Belyaeva et al., 2011; Serkin et al., 2010a;b). The concept of nonautonomous solitons, the generalized Lax pair and generalized AKNS methods described in details in this Chapter can be applied to different physical systems, from hydrodynamics and plasma physics to nonlinear optics and matter-waves and offer many opportunities for further scientific studies. As an illustrative example, we show that important mathematical analogies between different physical systems open the possibility to study optical rogue waves and ocean rogue waves in parallel and, due to the evident complexity of experiments with rogue waves in open oceans, this method offers remarkable possibilities in studies nonlinear hydrodynamic problems by performing experiments in the nonlinear optical systems with nonautonomous solitons and optical rogue waves.

\section{Lax operator method and exact integrability of nonautonomous nonlinear and dispersive models with external potentials}

The classification of dynamic systems into autonomous and nonautonomous is commonly used in science to characterize different physical situations in which, respectively, external time-dependent driving force is being present or absent. The mathematical treatment of nonautonomous system of equations is much more complicated then of traditional autonomous ones. As a typical illustration we may mention both a simple pendulum whose length changes with time and parametrically driven nonlinear Duffing oscillator (Nayfeh \& Balachandran, 2004).

In the framework of the IST method, the nonlinear integrable equation arises as the compatibility condition of the system of the linear matrix differential equations

$$
\psi_{x}=\widehat{\mathcal{F}} \psi(x, t), \quad \psi_{t}=\widehat{\mathcal{G}} \psi(x, t) .
$$


Here $\psi(x, t)=\left\{\psi_{1}, \psi_{2}\right\}^{T}$ is a 2-component complex function, $\widehat{\mathcal{F}}$ and $\widehat{\mathcal{G}}$ are complex-valued $(2 \times 2)$ matrices. Let us consider the general case of the IST method with a time-dependent spectral parameter $\Lambda(T)$ and the matrices $\widehat{\mathcal{F}}$ and $\widehat{\mathcal{G}}$

$$
\begin{aligned}
& \widehat{\mathcal{F}}(\Lambda ; S, T)=\widehat{\mathcal{F}}\left\{\Lambda(T), q[S(x, t), T] ; \frac{\partial q}{\partial S}\left(\frac{\partial S}{\partial x}\right) ; \frac{\partial^{2} q}{\partial S^{2}}\left(\frac{\partial S}{\partial x}\right)^{2} ; \ldots ; \frac{\partial^{n} q}{\partial S^{n}}\left(\frac{\partial S}{\partial x}\right)^{n}\right\} \\
& \widehat{\mathcal{G}}(\Lambda ; S, T)=\widehat{\mathcal{G}}\left\{\Lambda(T), q[S(x, t), T] ; \frac{\partial q}{\partial S}\left(\frac{\partial S}{\partial x}\right) ; \frac{\partial^{2} q}{\partial S^{2}}\left(\frac{\partial S}{\partial x}\right)^{2} ; \ldots ; \frac{\partial^{n} q}{\partial S^{n}}\left(\frac{\partial S}{\partial x}\right)^{n}\right\},
\end{aligned}
$$

dependent on the generalized coordinates $S=S(x, t)$ and $T(t)=t$, where the function $q[S(x, t), T]$ and its derivatives denote the scattering potentials $Q(S, T)$ and $R(S, T)$ and their derivatives, correspondingly. The condition for the compatibility of the pair of linear differential equations (1) takes a form

$$
\frac{\partial \widehat{\mathcal{F}}}{\partial T}+\frac{\partial \widehat{\mathcal{F}}}{\partial S} S_{t}-\frac{\partial \widehat{\mathcal{G}}}{\partial S} S_{x}+[\widehat{\mathcal{F}}, \widehat{\mathcal{G}}]=0
$$

where

$$
\begin{gathered}
\widehat{\mathcal{F}}=-i \Lambda(T) \widehat{\sigma_{3}}+\widehat{\mathcal{U}} \widehat{\phi}, \\
\widehat{\mathcal{G}}=\left(\begin{array}{cc}
A & B \\
C & -A
\end{array}\right),
\end{gathered}
$$

$\widehat{\sigma_{3}}$ is the Pauli spin matrix and matrices $\widehat{\mathcal{U}}$ and $\widehat{\phi}$ are given by

$$
\begin{gathered}
\widehat{\mathcal{U}}=\sqrt{\sigma} F^{\gamma}(T)\left(\begin{array}{cc}
0 & Q(S, T) \\
R(S, T) & 0
\end{array}\right), \\
\widehat{\phi}=\left(\begin{array}{cc}
\exp [-i \varphi / 2] & 0 \\
0 & \exp [i \varphi / 2]
\end{array}\right) .
\end{gathered}
$$

Here $F(T)$ and $\varphi(S, T)$ are real unknown functions, $\gamma$ is an arbitrary constant, and $\sigma= \pm 1$. The desired elements of $\widehat{\mathcal{G}}$ matrix (known in the modern literature as the AKNS elements) can be constructed in the form $\widehat{\mathcal{G}}=\sum_{k=0}^{k=3} G_{k} \Lambda^{k}$, with time varying spectral parameter given by

$$
\Lambda_{T}=\lambda_{0}(T)+\lambda_{1}(T) \Lambda(T),
$$

where time-dependent functions $\lambda_{0}(T)$ and $\lambda_{1}(T)$ are the expansion coefficients of $\Lambda_{T}$ in powers of the spectral parameter $\Lambda(T)$.

Solving the system (2-6), we find both the matrix elements $A, B, C$

$$
\begin{aligned}
A= & -i \lambda_{0} S / S_{x}+a_{0}-\frac{1}{4} a_{3} \sigma F^{2 \gamma}\left(Q R \varphi_{S} S_{x}+i Q R_{S} S_{x}-i R Q_{S} S_{x}\right) \\
& +\frac{1}{2} a_{2} \sigma F^{2 \gamma} Q R+\Lambda\left(-i \lambda_{1} S / S_{x}+\frac{1}{2} a_{3} \sigma F^{2 \gamma} Q R+a_{1}\right)+a_{2} \Lambda^{2}+a_{3} \Lambda^{3}, \\
B= & \sqrt{\sigma} F^{\gamma} \exp [i \varphi S / 2]\left\{-\frac{i}{4} a_{3} S_{x}^{2}\left(Q_{S S}+\frac{i}{2} Q \varphi_{S S}-\frac{1}{4} Q \varphi_{S}^{2}+i Q_{S} \varphi_{S}\right)\right. \\
& -\frac{i}{4} a_{2} Q \varphi_{S} S_{x}-\frac{1}{2} a_{2} Q_{S} S_{x}+i Q\left(-i \lambda_{1} S / S_{x}+\frac{1}{2} a_{3} \sigma F^{2 \gamma} Q R+a_{1}\right) \\
& \left.+\Lambda\left(-\frac{i}{4} a_{3} Q \varphi_{S} S_{x}-\frac{1}{2} a_{3} Q_{S} S_{x}+i a_{2} Q\right)+i a_{3} \Lambda^{2} Q\right\}
\end{aligned}
$$




$$
\begin{aligned}
C= & \sqrt{\sigma} F^{\gamma} \exp [-i \varphi S / 2]\left\{-\frac{i}{4} a_{3} S_{x}^{2}\left(R_{S S}-\frac{i}{2} R \varphi_{S S}-\frac{1}{4} R \varphi_{S}^{2}-i R_{S} \varphi_{S}\right)\right. \\
& -\frac{i}{4} a_{2} R \varphi_{S} S_{x}+\frac{1}{2} a_{2} R_{S} S_{x}+i R\left(-i \lambda_{1} S / S_{x}+\frac{1}{2} a_{3} \sigma F^{2 \gamma} Q R+a_{1}\right) \\
& \left.+\Lambda\left(-\frac{i}{4} a_{3} R \varphi_{S} S_{x}+\frac{1}{2} a_{3} R_{S} S_{x}+i a_{2} R\right)+i a_{3} \Lambda^{2} R\right\}
\end{aligned}
$$

and two general equations

$$
\begin{aligned}
& i Q_{T}=\frac{1}{4} a_{3} Q_{S S S} S_{x}^{3}+\frac{3 i}{8} a_{3} Q_{S S} \varphi_{S} S_{x}^{3}-\frac{3 i}{4} a_{3} \sigma F^{2 \gamma} Q^{2} R \varphi_{S} S_{x} \\
& -\frac{3}{2} a_{3} \sigma F^{2 \gamma} Q R Q_{S} S_{x}-\frac{i}{2} a_{2} Q_{S S} S_{x}^{2}+i a_{2} \sigma F^{2 \gamma} Q^{2} R \\
& +i Q_{S}\left(-S_{t}+\lambda_{1} S+i a_{1} S_{x}-\frac{i}{2} a_{2} \varphi_{S} S_{x}^{2}+\frac{3}{8} a_{3} \varphi_{S S} S_{x}^{3}+\frac{3 i}{16} a_{3} \varphi_{S}^{2} S_{x}^{3}\right) \\
& +Q\left(i \lambda_{1}-i \gamma \frac{F_{T}}{F}+\frac{1}{2} a_{2} \varphi_{S S} S_{x}^{2}-\frac{3}{16} a_{3} \varphi_{S} \varphi_{S S} S_{x}^{3}\right) \\
& +Q\left[2 \lambda_{0} S / S_{x}+2 i a_{0}+\frac{1}{2}\left(\varphi_{T}+\varphi_{S} S_{t}\right)-\frac{1}{2} \lambda_{1} S \varphi_{S}-\frac{i}{2} a_{1} \varphi_{S} S_{x}\right] \\
& +Q\left(\frac{i}{8} a_{2} \varphi_{S}^{2} S_{x}^{2}-\frac{i}{32} a_{3} \varphi_{S}^{3} S_{x}^{3}+\frac{i}{8} a_{3} \varphi_{S S S} S_{x}^{3}\right) \\
& i R_{T}=\frac{1}{4} a_{3} R_{S S S} S_{x}^{3}-\frac{3 i}{8} a_{3} R_{S S} \varphi_{S} S_{x}^{3}+\frac{3 i}{4} a_{3} \sigma F^{2 \gamma} R^{2} Q \varphi_{S} S_{x} \\
& -\frac{3}{2} a_{3} \sigma F^{2 \gamma} R^{2} Q_{S} S_{x}+\frac{i}{2} a_{2} R_{S S} S_{x}^{2}-i a_{2} \sigma F^{2 \gamma} R^{2} Q \\
& +i R_{S}\left(-S_{t}+\lambda_{1} S+i a_{1} S_{x}-\frac{i}{2} a_{2} \varphi_{S} S_{x}^{2}-\frac{3}{8} a_{3} \varphi_{S S} S_{x}^{3}+\frac{3 i}{16} a_{3} \varphi_{S}^{2} S_{x}^{3}\right) \\
& +R\left(i \lambda_{1}-i \gamma \frac{F_{T}}{F}+\frac{1}{2} a_{2} \varphi_{S S} S_{x}^{2}-\frac{3}{16} a_{3} \varphi_{S} \varphi_{S S} S_{x}^{3}\right) \\
& +R\left[-2 \lambda_{0} S / S_{x}-2 i a_{0}-\frac{1}{2}\left(\varphi_{T}+\varphi_{S} S_{t}\right)+\frac{1}{2} \lambda_{1} S \varphi_{S}+\frac{i}{2} a_{1} \varphi_{S} S_{x}\right] \\
& +R\left(-\frac{i}{8} a_{2} \varphi_{S}^{2} S_{x}^{2}+\frac{i}{32} a_{3} \varphi_{S}^{3} S_{x}^{3}-\frac{i}{8} a_{3} \varphi_{S S S} S_{x}^{3}\right),
\end{aligned}
$$

where the arbitrary time-dependent functions $a_{0}(T), a_{1}(T), a_{2}(T), a_{3}(T)$ have been introduced within corresponding integrations.

By using the following reduction procedure $R=-Q^{*}$, it is easy to find that two equations (9) and (10) take the same form if the following conditions

$$
\begin{array}{ll}
a_{0}=-a_{0}^{*}, & a_{1}=-a_{1}^{*}, \quad a_{2}=-a_{2}^{*}, \quad a_{3}=-a_{3}^{*}, \\
\lambda_{0}=\lambda_{0}^{*}, & \lambda_{1}=\lambda_{1}^{*}, \quad F=F^{*}
\end{array}
$$

are fulfilled. 


\section{Generalized nonlinear Schrödinger equation and solitary waves in nonautonomous nonlinear and dispersive systems: nonautonomous solitons}

Let us study a special case of the reduction procedure for Eqs. $(9,10)$ when $a_{3}=0$

$$
\begin{aligned}
& A=-i \lambda_{0} S / S_{x}+a_{0}(T)-\frac{1}{2} a_{2}(T) \sigma F^{2 \gamma}|Q|^{2}-i \lambda_{1} S / S_{x} \Lambda+a_{1}(T) \Lambda+a_{2}(T) \Lambda^{2}, \\
& B=\sqrt{\sigma} F^{\gamma} \exp (i \varphi / 2)\left\{-\frac{i}{4} a_{2}(T) Q \varphi_{S} S_{x}-\frac{1}{2} a_{2}(T) Q_{S} S_{x}\right\}+ \\
& i\left\{Q\left[-i \lambda_{1} S / S_{x}+a_{1}(T)+\Lambda a_{2}(T)\right]\right\}, \\
& C= \sqrt{\sigma} F^{\gamma} \exp (-i \varphi / 2)\left\{\frac{i}{4} a_{2}(T) Q^{*} \varphi_{S} S_{x}-\frac{1}{2} a_{2}(T) Q_{S}^{*} S_{x}\right\} \\
&-i\left\{Q^{*}\left[-i \lambda_{1} x+a_{1}(T)+\Lambda a_{2}(T)\right]\right\} .
\end{aligned}
$$

In accordance with conditions (11), the imaginary functions $a_{0}(T), a_{1}(T), a_{2}(T)$ can be defined in the following way: $a_{0}(T)=i \gamma_{0}(T), a_{1}(T)=i V(T), a_{2}(T)=-i D_{2}(T), R_{2}(T)=$ $F^{2 \gamma} D_{2}(T)$, where $D_{2}(T), V(T), \gamma_{0}(T)$ are arbitrary real functions. The coefficients $D_{2}(T)$ and $R_{2}(T)$ are represented by positively defined functions (for $\sigma=-1, \gamma$ is assumed as a semi-entire number).

Then, Eqs. $(9,10)$ can be transformed into

$$
i Q_{T}=-\frac{1}{2} D_{2} Q_{S S} S_{x}^{2}-\sigma R_{2}|Q|^{2} Q-i \widetilde{V} Q_{S}+i \Gamma Q+U Q,
$$

where

$$
\begin{gathered}
\widetilde{V}(S, T)=\frac{1}{2} D_{2} S_{x}^{2} \varphi_{S}+V S_{x}+S_{t}-\lambda_{1} S, \\
U(S, T)=\frac{1}{8} D_{2} S_{x}^{2} \varphi_{S}^{2}-2 \gamma_{0}+\frac{1}{2}\left(\varphi_{T}+\varphi_{S} S_{t}+V S_{x} \varphi_{S}\right)+2 \lambda_{0} S / S_{x}-\frac{1}{2} \lambda_{1} \varphi_{S} S, \\
\Gamma=\left(-\gamma \frac{F_{T}}{F}-\frac{1}{4} D_{2} S_{x}^{2} \varphi_{S S}+\lambda_{1}\right)=\left(\frac{1}{2} \frac{W\left(R_{2}, D_{2}\right)}{R_{2} D_{2}}-\frac{1}{4} D_{2} S_{x}^{2} \varphi_{S S}+\lambda_{1}\right) .
\end{gathered}
$$

Eq.(12) can be written down in the independent variables $(x, t)$

$$
i Q_{t}+\frac{1}{2} D_{2}(t) Q_{x x}+\sigma R_{2}(t)|Q|^{2} Q-U(x, t) Q+i \widetilde{V^{\prime}} Q_{x}=i \Gamma(t) Q .
$$

Let us transform Eq.(15) into the more convenient form

$$
i Q_{t}+\frac{1}{2} D_{2} Q_{x x}+\sigma R_{2}|Q|^{2} Q-U Q=i \Gamma Q
$$

using the following condition

$$
\widetilde{V^{\prime}}=\frac{1}{2} D_{2} S_{x} \varphi_{S}+V-\lambda_{1} S / S_{x}=0 .
$$

If we apply the commonly accepted in the IST method (Ablowitz et al., 1973) reduction: $V=$ $-i a_{1}=0$, we find a parameter $\lambda_{1}$ from (17)

$$
\lambda_{1}=\frac{1}{2} D_{2} S_{x}^{2} \varphi_{S} / S
$$


and the corresponding potential $U(S, T)$ from Eq.(13):

$$
U(S, T)=-2 \gamma_{0}+2 \lambda_{0} S / S_{x}+\frac{1}{2}\left(\varphi_{T}+\varphi_{S} S_{t}\right)-\frac{1}{8} D_{2} S_{x}^{2} \varphi_{S}^{2}
$$

According to Eq.(14), the gain or absorption coefficient now is represented by

$$
\Gamma=\frac{1}{2} \frac{W\left(R_{2}, D_{2}\right)}{R_{2} D_{2}}-\frac{1}{4} D_{2} S_{x}^{2} \varphi_{S S}+\frac{1}{2} D_{2} S_{x}^{2} \varphi_{S} / S .
$$

Let us consider some special choices of variables to specify the solutions of (16). First of all, we assume that variables are factorized in the phase profile $\varphi(S, T)$ as $\varphi=C(T) S^{\alpha}$. The first term in the real potential (19) represents some additional time-dependent phase $e^{2 \gamma_{0}(t) t}$ of the solution $Q(x, t)$ for the equation (16) and, without loss of the generality, we use $\gamma_{0}=0$. The second term in (19) depends linearly on $S$. The NLSE with the linear spatial potential and constant $\lambda_{0}$, describing the case of Alfen waves propagation in plasmas, has been studied previously in Ref. (Chen, 1976). We will study the more general case of chirped solitons in the Section 4 of this Chapter. Now, taking into account three last terms in (19), we obtain

$$
U(S, T)=2 \lambda_{0} S / S_{x}+\frac{1}{2} C_{T} S^{\alpha}+1 / 2 \alpha C S^{\alpha-1} S_{t}-\frac{1}{8} D_{2} C^{2} S_{x}^{2} \alpha^{2} S^{2 \alpha-2} .
$$

The gain or absorption coefficient (20) becomes

$$
\Gamma(T)=\frac{1}{2} \frac{W\left(R_{2}, D_{2}\right)}{R_{2} D_{2}}+\frac{\alpha}{4}(3-\alpha) D_{2} S_{x}^{2} C S^{\alpha-2}
$$

and Eq.(18) takes a form

$$
\lambda_{1}=\frac{1}{2} D_{2} S_{x}^{2} C \alpha S^{\alpha-2} .
$$

If we assume that the functions $\Gamma(T)$ and $\lambda_{1}(T)$ depend only on $T$ and do not depend on $S$, we conclude that $\alpha=0$ or $\alpha=2$.

The study of the soliton solutions of the nonautonomous NLSE with varying coefficients without time and space phase modulation (chirp) and corresponding to the case of $\alpha=0$ has been carried out in Ref. (Serkin \& Belyaeva, 2001a;b). Let us find here the solutions of Eq.(16) with chirp in the case of $\alpha=2, \varphi(S, T)=C(T) S^{2}$. In this case, Eq. (18) becomes $\lambda_{1}=D_{2} S_{x}^{2} C$. Now, the real spatial-temporal potential (21) takes the form

$$
U[S(x, t), T)]=2 \lambda_{0} S / S_{x}+\frac{1}{2}\left(C_{T}-D_{2} S_{x}^{2} C^{2}\right) S^{2}+C S S_{t}
$$

Consider the simplest option to choose the variable $S(x, t)$ when the variables $(x, t)$ are factorized: $S(x, t)=P(t) x$. In this case, all main characteristic functions: the phase modulation

the real potential

$$
\varphi(x, t)=\Theta(t) x^{2},
$$

$$
U(x, t)=2 \lambda_{0} x+\frac{1}{2}\left(\Theta_{t}-D_{2} \Theta^{2}\right) x^{2} \equiv 2 \lambda_{0}(t) x+\frac{1}{2} \Omega^{2}(t) x^{2},
$$

the gain (or absorption) coefficient

$$
\Gamma(t)=\frac{1}{2}\left(\frac{W\left(R_{2}, D_{2}\right)}{R_{2} D_{2}}+D_{2} P^{2} C\right)=\frac{1}{2}\left(\frac{W\left(R_{2}, D_{2}\right)}{R_{2} D_{2}}+D_{2} \Theta\right)
$$


and the spectral parameter $\lambda_{1}$

$$
\lambda_{1}(t)=D_{2} P^{2} C=D_{2}(t) \Theta(t)
$$

are found to be dependent on the self-induced soliton phase shift $\Theta(t)$. Notice that the definition $\Omega^{2}(t) \equiv \Theta_{t}-D_{2} \Theta^{2}$ has been introduced in Eq.(25).

Now we can rewrite the generalized NLSE (16) with time-dependent nonlinearity, dispersion and gain or absorption in the form of the nonautonomous NLSE with linear and parabolic potentials

$$
i Q_{t}+\frac{1}{2} D_{2}(t) Q_{x x}+\sigma R_{2}(t)|Q|^{2} Q-2 \lambda_{0}(t) x-\frac{1}{2} \Omega^{2}(t) x^{2} Q=i \Gamma Q .
$$

\section{Hidden features of the soliton adaptation law to external potentials: the generalized Serkin-Hasegawa theorems}

It is now generally accepted that solitary waves in nonautonomous nonlinear and dispersive systems can propagate in the form of so-called nonautonomous solitons or solitonlike similaritons (see (Atre et al., 2006; Avelar et al., 2009; Belić et al., 2008; Chen et al., 2007; Hao, 2008; He et al., 2009; Hernandez et al., 2005; Hernandez-Tenorio et al., 2007; Liu et al., 2008; Porsezian et al., 2009; 2007; Serkin et al., 2007; Shin, 2008; Tenorio et al., 2005; Wang et al., 2008; Wu, Li \& Zhang, 2008; Wu, Zhang, Li, Finot \& Porsezian, 2008; Zhang et al., 2008; Zhao et al., 2009; 2008) and references therein). Nonautonomous solitons interact elastically and generally move with varying amplitudes, speeds and spectra adapted both to the external potentials and to the dispersion and nonlinearity variations. The existence of specific laws of soliton adaptation to external gain and loss potentials was predicted by Serkin and Hasegawa in 2000 (Serkin \& Hasegawa, 2000a;b; 2002). The physical mechanism resulting in the soliton stabilization in nonautonomous and dispersive systems was revealed in this paper. From the physical point of view, the adaptation means that solitons remain self similar and do not emit dispersive waves both during their interactions with external potentials and with each other. The soliton adaptation laws are known today as the Serkin-Hasegawa theorems (SH theorems). Serkin and Hasegawa obtained their SH-theorems by using the symmetry reduction methods when the initial nonautonomous NLSE can be transformed by the canonical autonomous NLSE under specific conditions found in (Serkin \& Hasegawa, 2000a;b). Later, SH-theorems have been confirmed by different methods, in particular, by the Painleve analysis and similarity transformations (Serkin \& Hasegawa, 2000a;b; 2002; Serkin et al., 2004; 2007; 2001a;b).

Substituting the phase profile $\Theta(t)$ given by Eq. (26) into Eq. (25), it is straightforward to verify that the frequency of the harmonic potential $\Omega(t)$ is related with dispersion $D_{2}(t)$, nonlinearity $R_{2}(t)$ and gain or absorption coefficient $\Gamma(t)$ by the following conditions

$$
\begin{aligned}
& \Omega^{2}(t) D_{2}(t)= D_{2}(t) \frac{d}{d t}\left(\frac{\Gamma(t)}{D_{2}(t)}\right)-\Gamma^{2}(t) \\
&-\frac{d}{d t}\left(\frac{W\left(R_{2}, D_{2}\right)}{R_{2} D_{2}}\right)+\left(2 \Gamma(t)+\frac{d}{d t} \ln R_{2}(t)\right) \frac{W\left(R_{2}, D_{2}\right)}{R_{2} D_{2}} \\
&=D_{2}(t) \frac{d}{d t}\left(\frac{\Gamma(t)}{D_{2}(t)}\right)-\Gamma^{2}(t)+\left(2 \Gamma(t)+\frac{d}{d t} \ln R_{2}(t)\right) \frac{d}{d t} \ln \frac{D_{2}(t)}{R_{2}(t)}-\frac{d^{2}}{d t^{2}} \ln \frac{D_{2}(t)}{R_{2}(t)},
\end{aligned}
$$

where $W\left(R_{2}, D\right)=R_{2} D_{2 t}^{\prime}-D_{2} R_{2 t}^{\prime}$ is the Wronskian. 
After the substitutions

$$
Q(x, t)=q(x, t) \exp \left[\int_{0}^{t} \Gamma(\tau) d \tau\right], R(t)=R_{2}(t) \exp \left[2 \int_{0}^{t} \Gamma(\tau) d \tau\right], D(t)=D_{2}(t),
$$

Eq. (28) is transformed to the generalized NLSE without gain or loss term

$$
i \frac{\partial q}{\partial t}+\frac{1}{2} D(t) \frac{\partial^{2} q}{\partial x^{2}}+\left[\sigma R(t)|q|^{2}-2 \lambda_{0}(t) x-\frac{1}{2} \Omega^{2}(t) x^{2}\right] q=0 .
$$

Finally, the Lax equation (2) with matrices (3-6) provides the nonautonomous model (30) under condition that dispersion $D(t)$, nonlinearity $R(t)$, and the harmonic potential satisfy to the following exact integrability conditions

$$
\begin{aligned}
\Omega^{2}(t) D(t) & =\frac{W(R, D)}{R D} \frac{d}{d t} \ln R(t)-\frac{d}{d t}\left(\frac{W(R, D)}{R D}\right) \\
& =\frac{d}{d t} \ln D(t) \frac{d}{d t} \ln R(t)-\frac{d^{2}}{d t^{2}} \ln D(t)-R(t) \frac{d^{2}}{d t^{2}} \frac{1}{R(t)} .
\end{aligned}
$$

The self-induced soliton phase shift is given by

$$
\Theta(t)=-\frac{W[(R(t), D(t)]}{D^{2}(t) R(t)}
$$

and the time-dependent spectral parameter is represented by

$$
\Lambda(t)=\kappa(t)+i \eta(t)=\frac{D_{0} R(t)}{R_{0} D(t)}\left[\Lambda(0)+\frac{R_{0}}{D_{0}} \int_{0}^{t} \frac{\lambda_{0}(\tau) D(\tau)}{R(\tau)} d \tau\right]
$$

where the main parameters: time invariant eigenvalue $\Lambda(0)=\kappa_{0}+i \eta_{0} ; D_{0}=D(0) ; R_{0}=$ $R(0)$ are defined by the initial conditions.

We call Eq. (31) as the law of the soliton adaptation to the external potentials. The basic property of classical solitons to interact elastically holds true, but the novel feature of the nonautonomous solitons arises. Namely, both amplitudes and speeds of the solitons, and consequently, their spectra, during the propagation and after the interaction are no longer the same as those prior to the interaction. All nonautonomous solitons generally move with varying amplitudes $\eta(t)$ and speeds $\kappa(t)$ adapted both to the external potentials and to the dispersion $D(t)$ and nonlinearity $R(t)$ changes.

Having obtained the eigenvalue equations for scattering potential, we can write down the general solutions for bright $(\sigma=+1)$ and dark $(\sigma=-1)$ nonautonomous solitons applying the auto-Bäcklund transformation (Chen, 1974) and the recurrent relation

$$
q_{n}(x, t)=-q_{n-1}(x, t)-\frac{4 \eta_{n} \widetilde{\boldsymbol{\Gamma}}_{n-1}(x, t)}{1+\left|\widetilde{\boldsymbol{\Gamma}}_{n-1}(x, t)\right|^{2}} \times \sqrt{\frac{D(t)}{R(t)}} \exp \left[-i \Theta x^{2} / 2\right],
$$

which connects the $(n-1)$ and $n$ - soliton solutions by means of the so-called pseudo-potential $\widetilde{\boldsymbol{\Gamma}}_{n-1}(x, t)=\psi_{1}(x, t) / \psi_{2}(x, t)$ for the $(n-1)$-soliton scattering functions $\psi(x, t)=\left(\psi_{1} \psi_{2}\right)^{T}$. 
Bright $q_{1}^{+}(x, t)$ and dark $q_{1}^{-}(x, t)$ soliton solutions are represented by the following analytic expressions:

$$
\begin{aligned}
& q_{1}^{+}(x, t \mid \sigma=+1)= 2 \eta_{1}(t) \sqrt{\frac{D(t)}{R(t)}} \operatorname{sech}\left[\xi_{1}(x, t)\right] \times \exp \left\{-i\left(\frac{\Theta(t)}{2} x^{2}+\chi_{1}(x, t)\right)\right\} ; \\
& q_{1}^{-}(x, t \mid \sigma=-1)=2 \eta_{1}(t) \sqrt{\frac{D(t)}{R(t)}}\left[\sqrt{\left(1-a^{2}\right)}+i a \tanh \zeta(x, t)\right] \\
& \times \exp \left\{-i\left(\frac{\Theta(t)}{2} x^{2}+\phi(x, t)\right)\right\}, \\
& \zeta(x, t)=2 a \eta_{1}(t) x+4 a \int_{0}^{t} D(\tau) \eta_{1}(\tau) \kappa_{1}(\tau) d \tau, \\
& \phi(x, t)=2\left[\kappa_{1}(t)-\eta_{1}(t) \sqrt{\left(1-a^{2}\right)}\right] x \\
&+2 \int_{0}^{t} D(\tau)\left[\kappa_{1}^{2}+\eta_{1}^{2}\left(3-a^{2}\right)-2 \kappa_{1} \eta_{1} \sqrt{\left(1-a^{2}\right)}\right] d \tau .
\end{aligned}
$$

Dark soliton (36) has an additional parameter, $0 \leq a \leq 1$, which designates the depth of modulation (the blackness of gray soliton) and its velocity against the background. When $a=1$, dark soliton becomes black. For optical applications, Eq.(36) can be easily transformed into the Hasegawa and Tappert form for the nonautonomous dark solitons (Hasegawa, 1995) under the condition $\kappa_{0}=\eta_{0} \sqrt{\left(1-a^{2}\right)}$ that corresponds to the special choice of the retarded frame associated with the group velocity of the soliton

$$
\begin{gathered}
q_{1}^{-}(x, t \mid \sigma=-1)=2 \eta_{1}(t) \sqrt{\frac{D(t)}{R(t)}}\left[\sqrt{\left(1-a^{2}\right)}+i a \tanh \widetilde{\zeta}(x, t)\right] \\
\times \exp \left\{-i\left(\frac{\Theta(t)}{2} x^{2}+\widetilde{\phi}(x, t)\right)\right\}, \\
\widetilde{\zeta}(x, t)=2 a \eta_{1}(t) x+4 a \int_{0}^{t} D(\tau) \eta_{1}(\tau)\left[\eta_{1}(\tau) \sqrt{\left(1-a^{2}\right)}+K(\tau)\right] d \tau, \\
\widetilde{\phi}(x, t)=2 K(t) x+2 \int_{0}^{t} D(\tau)\left[K^{2}(\tau)+2 \eta_{1}^{2}(\tau)\right] d \tau, \\
K(t)=\frac{R(t)}{D(t)} \int_{0}^{t} \lambda_{0}(\tau) \frac{D(\tau)}{R(\tau)} d \tau .
\end{gathered}
$$

Notice that the solutions considered here hold only when the nonlinearity, dispersion and confining harmonic potential are related by Eq. (31), and both $D(t) \neq 0$ and $R(t) \neq 0$ for all times by definition. 
Two-soliton $q_{2}(x, t)$ solution for $\sigma=+1$ follows from Eq. (34)

$$
q_{2}(x, t)=4 \sqrt{\frac{D(t)}{R(t)}} \frac{\mathrm{N}(x, t)}{\mathrm{D}(x, t)} \exp \left[-\frac{i}{2} \Theta(t) x^{2}\right],
$$

where the numerator $\mathrm{N}(x, t)$ is given by

$$
\begin{aligned}
\mathrm{N}= & \cosh \xi_{2} \exp \left(-i \chi_{1}\right) \\
& \times\left[\left(\kappa_{2}-\kappa_{1}\right)^{2}+2 i \eta_{2}\left(\kappa_{2}-\kappa_{1}\right) \tanh \xi_{2}+\eta_{1}^{2}-\eta_{2}^{2}\right]+\eta_{2} \cosh \xi_{1} \exp \left(-i \chi_{2}\right) \\
& \times\left[\left(\kappa_{2}-\kappa_{1}\right)^{2}-2 i \eta_{1}\left(\kappa_{2}-\kappa_{1}\right) \tanh \xi_{1}-\eta_{1}^{2}+\eta_{2}^{2}\right],
\end{aligned}
$$

and the denominator $\mathrm{D}(x, t)$ is represented by

$$
\begin{aligned}
\mathrm{D}= & \cosh \left(\xi_{1}+\xi_{2}\right)\left[\left(\kappa_{2}-\kappa_{1}\right)^{2}+\left(\eta_{2}-\eta_{1}\right)^{2}\right] \\
& +\cosh \left(\xi_{1}-\xi_{2}\right)\left[\left(\kappa_{2}-\kappa_{1}\right)^{2}+\left(\eta_{2}+\eta_{1}\right)^{2}\right]-4 \eta_{1} \eta_{2} \cos \left(\chi_{2}-\chi_{1}\right) .
\end{aligned}
$$

Arguments and phases in Eqs.(39-41)

$$
\begin{aligned}
& \xi_{i}(x, t)=2 \eta_{i}(t) x+4 \int_{0}^{t} D(\tau) \eta_{i}(\tau) \kappa_{i}(\tau) d \tau \\
& \chi_{i}(x, t)=2 \kappa_{i}(t) x+2 \int_{0}^{t} D(\tau)\left[\kappa_{i}^{2}(\tau)-\eta_{i}^{2}(\tau)\right] d \tau
\end{aligned}
$$

are related with the amplitudes

$$
\eta_{i}(t)=\frac{D_{0} R(t)}{R_{0} D(t)} \eta_{0 i}
$$

and velocities

$$
\kappa_{i}(t)=\frac{D_{0} R(t)}{R_{0} D(t)}\left[\kappa_{0 i}+\frac{R_{0}}{D_{0}} \int_{0}^{t} \frac{\lambda_{0}(\tau) D(\tau)}{R(\tau)} d \tau\right]
$$

of the nonautonomous solitons, where $\kappa_{0 i}$ and $\eta_{0 i}$ correspond to the initial velocity and amplitude of the $i$-th soliton $(i=1,2)$.

Eqs. (39-45) describe the dynamics of two bounded solitons at all times and all locations. Obviously, these soliton solutions reduce to classical soliton solutions in the limit of autonomous nonlinear and dispersive systems given by conditions: $R(t)=D(t)=1$, and $\lambda_{0}(t)=\Omega(t) \equiv 0$ for canonical NLSE without external potentials. 


\section{Chirped optical solitons with moving spectra in nonautonomous systems: colored nonautonomous solitons}

Both the nonlinear Schrödinger equations $(28,30)$ and the Lax pair equations $(3-6)$ are written down here in the most general form. The transition to the problems of optical solitons is accomplished by the substitution $x \rightarrow T$ (or $x \rightarrow X) ; t \rightarrow Z$ and $q^{+}(x, t) \rightarrow \widetilde{u}^{+}(Z, T($ or $X)$ ) for bright solitons, and $\left[q^{-}(x, t)\right]^{*} \rightarrow \widetilde{u}^{-}(Z, T($ or $X))$ for dark solitons, where the asterisk denotes the complex conjugate, $Z$ is the normalized distance, and $T$ is the retarded time for temporal solitons, while $X$ is the transverse coordinate for spatial solitons.

The important special case of Eq.(30) arises under the condition $\Omega^{2}(Z)=0$. Let us rewrite Eq. (30) by using the reduction $\Omega=0$, which denotes that the confining harmonic potential is vanishing

$$
i \frac{\partial u}{\partial Z}+\frac{\sigma}{2} D(Z) \frac{\partial^{2} u}{\partial T^{2}}+R(Z)|u|^{2} u-2 \sigma \lambda_{0}(Z) T u=0 .
$$

This implies that the self-induced soliton phase shift $\Theta(Z)$, dispersion $D(Z)$, and nonlinearity $R(Z)$ are related by the following law of soliton adaptation to external linear potential

$$
D(Z) / D_{0}=R(Z) / R_{0} \exp \left\{-\frac{\Theta_{0} D_{0}}{R_{0}} \int_{0}^{Z} R(\tau) d \tau\right\} .
$$

Nonautonomous exactly integrable NLSE model given by Eqs. $(46,47)$ can be considered as the generalization of the well-studied Chen and Liu model (Chen, 1976) with linear potential $\lambda_{0}(Z) \equiv \alpha_{0}=$ const and $D(Z)=D_{0}=R(Z)=R_{0}=1, \sigma=+1, \Theta_{0}=0$. It is interesting to note that the accelerated solitons predicted by Chen and Liu in plasma have been discovered in nonlinear fiber optics only decade later (Agrawal, 2001; Dianov et al., 1989; Taylor, 1992). Notice that nonautonomous solitons with nontrivial self-induced phase shifts and varying amplitudes, speeds and spectra for Eq. (46) are given in quadratures by Eqs. (35-45) under condition $\Omega^{2}(Z)=0$.

Let us show that the so-called Raman colored optical solitons can be approximated by this equation. Self-induced Raman effect (also called as soliton self-frequency shift) is being described by an additional term in the NLSE: $-\sigma_{R} U \partial|U|^{2} / \partial T$, where $\sigma_{R}$ originates from the frequency dependent Raman gain (Agrawal, 2001; Dianov et al., 1989; Taylor, 1992). Assuming that soliton amplitude does not vary significantly during self-scattering $|U|^{2}=\eta^{2} \operatorname{sech}^{2}(\eta T)$, we obtain that

$$
\sigma_{R} \frac{\partial|U|^{2}}{\partial T} \approx-2 \sigma_{R} \eta^{4} T=2 \alpha_{0} T
$$

and $d v / d Z=\sigma_{R} \eta^{4} / 2$, where $v=\kappa / 2$. The result of soliton perturbation theory (Agrawal, 2001; Dianov et al., 1989; Taylor, 1992) gives $d v / d Z=8 \sigma_{R} \eta^{4} / 15$. This fact explains the remarkable stability of colored Raman solitons that is guaranteed by the property of the exact integrability of the Chen and Liu model (Chen, 1976). More general model Eq. (46) and its exact soliton solutions open the possibility of designing an effective soliton compressor, for example, by drawing a fiber with $R(Z)=1$ and $D(Z)=\exp \left(-c_{0} Z\right)$, where $c_{0}=\Theta_{0} D_{0}$. It seems very attractive to use the results of nonautonomous solitons concept in ultrashort photonic applications and soliton lasers design.

Another interesting feature of the novel solitons, which we called colored nonautonomous solitons, is associated with the nontrivial dynamics of their spectra. Frequency spectrum of the chirped nonautonomous optical soliton moves in the frequency domain. In particular, 
if dispersion and nonlinearity evolve in unison $D(t)=R(t)$ or $D=R=1$, the solitons propagate with identical spectra, but with totally different time-space behavior.

Consider in more details the case when the nonlinearity $R=R_{0}$ stays constant but the dispersion varies exponentially along the propagation distance

$$
\begin{aligned}
& D(Z)=D_{0} \exp \left(-c_{0} Z\right), \\
& \Theta(Z)=\Theta_{0} \exp \left(c_{0} Z\right) .
\end{aligned}
$$

Let us write the one and two soliton solutions in this case with the lineal potential that, for simplicity, does not depend on time: $\lambda_{0}(Z)=\alpha_{0}=$ const

$$
\begin{gathered}
U_{1}(Z, T)=2 \eta_{01} \sqrt{D_{0} \exp \left(c_{0} Z\right)} \operatorname{sech}\left[\xi_{1}(Z, T)\right] \times \exp \left[-\frac{i}{2} \Theta_{0} \exp \left(c_{0} Z\right) T^{2}-i \chi_{1}(Z, T)\right], \\
U_{2}(Z, T)=4 \sqrt{D_{0} \exp \left(-c_{0} Z\right)} \frac{\mathrm{N}(Z, T)}{\mathrm{D}(Z, T)} \exp \left[-\frac{i}{2} \Theta_{0} \exp \left(c_{0} Z\right) T^{2}\right],
\end{gathered}
$$

where the nominator $\mathrm{N}(Z, T)$ and denominator $\mathrm{D}(Z, T)$ are given by Eqs. $(40,41)$ and

$$
\begin{aligned}
\xi_{i}(Z, T)= & 2 \eta_{0 i} T \exp \left(c_{0} Z\right)+4 D_{0} \eta_{0 i} \\
& \times\left\{\frac{\kappa_{0 i}}{c_{0}}\left[\exp \left(c_{0} Z\right)-1\right]+\frac{\alpha_{0}}{c_{0}}\left[\frac{\exp \left(c_{0} Z\right)-1}{c_{0}}-Z\right]\right\}, \\
\chi_{i}(Z, T)= & 2 \kappa_{0 i} T \exp \left(c_{0} Z\right)+2 D_{0}\left(\kappa_{0 i}^{2}-\eta_{0 i}^{2}\right) \frac{\exp \left(2 c_{0} Z\right)-1}{2 c_{0}} \\
& +2 T \frac{\alpha_{0}}{c_{0}}\left[\exp \left(c_{0} Z\right)-1\right]+4 D_{0} \kappa_{0 i} \frac{\alpha_{0}}{c_{0}}\left[\frac{\exp \left(c_{0} Z\right)-1}{c_{0}}-t\right] \\
& +2 D_{0}\left(\frac{\alpha_{0}}{c_{0}}\right)^{2}\left[\frac{\exp \left(c_{0} Z\right)-\exp \left(-c_{0} Z\right)}{c_{0}}-2 Z\right] .
\end{aligned}
$$

The initial velocity and amplitude of the $i$-th soliton $(i=1,2)$ are denoted by $\kappa_{0 i}$ and $\eta_{0 i}$.

We display in Fig.1 $(a, b)$ the main features of nonautonomous colored solitons to show not only their acceleration and reflection from the lineal potential, but also their compression and amplitude amplification. Dark soliton propagation and dynamics are presented in Fig.1(c,d). The limit case of the Eqs.(48-51) appears when $c_{0} \rightarrow \infty$ (that means $D(Z)=D_{0}=$ constant) and corresponds to the Chen and Liu model (Chen, 1976). The solitons with argument and phase

$$
\begin{aligned}
& \xi(Z, T)=2 \eta_{0}\left(T+2 \kappa_{0} Z+\alpha_{0} Z^{2}-T_{0}\right), \\
& \chi(Z, T)=2 \kappa_{0} T+2 \alpha_{0} T Z+2\left(\kappa_{0}^{2}-\eta_{0}^{2}\right) Z+2 \kappa_{0} \alpha_{0} Z^{2}+\frac{2}{3} \alpha_{0}^{2} Z^{3}
\end{aligned}
$$

represents the particle-like solutions which may be accelerated and reflected from the lineal potential. 


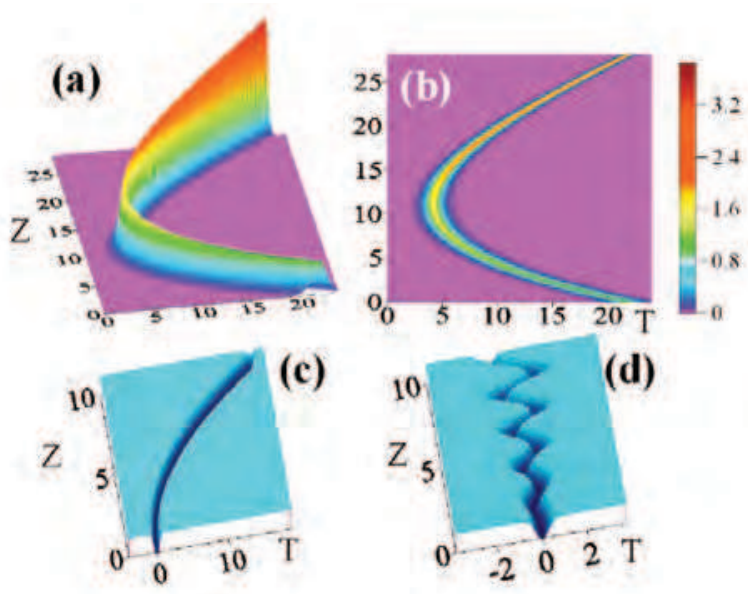

Fig. 1. Evolution of nonautonomous bright $(a, b)$ optical soliton calculated within the framework of the generalized model given by Eqs. (46-51) after choosing the soliton management parameters $c_{0}=0.05, \alpha_{0}=-0.2, \eta_{10}=0.5, \kappa_{10}=1.5$. (a) the temporal behavior; (b) the corresponding contour map. (c,d) Dark nonautonomous soliton dynamics within the framework of the model Eqs. $(46,47)$ after choosing the soliton management parameters: (c) $\mathrm{R}=-\mathrm{D}=1.0$ and $\alpha_{0}=-1.0$ and $(\mathrm{d}) \mathrm{R}=-\mathrm{D}=\cos (\omega \mathrm{Z})$, where $\omega=3.0$.

\section{Bound states of colored nonautonomous optical solitons: nonautonomous "agitated" breathers.}

Let us now give the explicit formula of the soliton solutions $(48,49)$ for the case where all eigenvalues are pure imaginary, or the initial velocities of the solitons are equal to zero. In the case $N=1$ and $\lambda_{0}(Z)=0$, we obtain

$$
\begin{aligned}
U_{1}(Z, T)=2 & \left.\eta_{01} \sqrt{D_{0} \exp \left(c_{0} Z\right)} \operatorname{sech}\left[2 \eta_{01} T \exp \left(c_{0} Z\right)\right)\right] \\
& \times \exp \left[-\frac{i}{2} \Theta_{0} \exp \left(c_{0} Z\right) T^{2}+i 2 D_{0} \eta_{01}^{2} \frac{\exp \left(2 c_{0} Z\right)-1}{2 c_{0}}\right] .
\end{aligned}
$$

This result shows that the laws of soliton adaptation to the external potentials (31) allow to stabilize the soliton even without a trapping potential. In addition, Eq.(52) indicates the possibility for the optimal compression of solitons, which is shown in Fig.2. We stress that direct computer experiment confirms the exponential in time soliton compression scenario in full accordance with analytical expression Eq.(52).

The bound two-soliton solution for the case of the pure imaginary eigenvalues is represented by

where

$$
U_{2}(Z, T)=4 \sqrt{D_{0} \exp \left(-c_{0} Z\right)} \frac{\mathrm{N}(Z, T)}{\mathrm{D}(Z, T)} \exp \left[-\frac{i}{2} \Theta_{0} \exp \left(c_{0} Z\right) T^{2}\right],
$$

$$
\begin{gathered}
\mathrm{N}=\left(\eta_{01}^{2}-\eta_{02}^{2}\right) \exp \left(c_{0} Z\right)\left[\eta_{01} \cosh \xi_{2} \exp \left(-i \chi_{1}\right)-\eta_{02} \cosh \xi_{1} \exp \left(-i \chi_{2}\right)\right], \\
\mathrm{D}=\cosh \left(\xi_{1}+\xi_{2}\right)\left(\eta_{01}-\eta_{02}\right)^{2}+\cosh \left(\xi_{1}-\xi_{2}\right)\left(\eta_{01}+\eta_{02}\right)^{2}-4 \eta_{01} \eta_{02} \cos \left(\chi_{2}-\chi_{1}\right),
\end{gathered}
$$




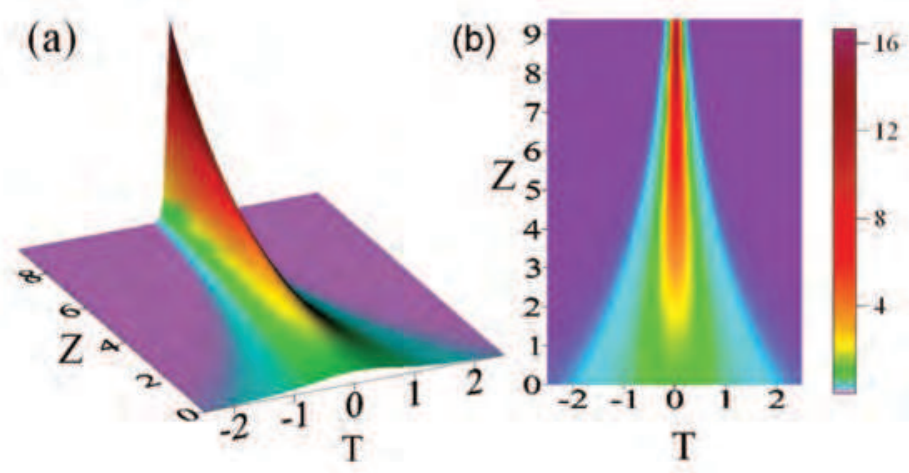

Fig. 2. Self-compression of nonautonomous soliton calculated within the framework of the model Eq. (46) after choosing the soliton management parameters $c_{0}=0.05 ; \alpha=0$ and $\eta_{0}=0.5$. (a) the temporal behavior; (b) the corresponding contour map.

and

$$
\begin{gathered}
\xi_{i}(Z, T)=2 \eta_{0 i} T \exp \left(c_{0} Z\right), \\
\chi_{i}(Z, T)=-2 D_{0} \eta_{0 i}^{2} \frac{\exp \left(2 c_{0} Z\right)-1}{2 c_{0}}+\chi_{i 0} .
\end{gathered}
$$

For the particular case of $\eta_{10}=1 / 2, \eta_{20}=3 / 2$ Eqs.(53-57) are transformed to

$$
\begin{aligned}
U_{2}(Z, T)= & 4 \sqrt{D_{0} \exp \left(-c_{0} Z\right)} \exp \left[-\frac{i}{2} \Theta_{0} \exp \left(c_{0} Z\right) T^{2}\right] \\
& \times \exp \left[\frac{i}{4 c_{0}} D_{0}\left[\exp \left(2 c_{0} Z\right)-1\right]+\chi_{10}\right] \\
& \times \frac{\cosh 3 X-3 \cosh X \exp \left\{i 2 D_{0}\left[\exp \left(2 c_{0} Z\right)-1\right] / c_{0}+i \Delta \varphi\right\}}{\cosh 4 X+4 \cosh 2 X-3 \cos \left\{2 D_{0}\left[\exp \left(2 c_{0} Z\right)-1\right] / c_{0}+\Delta \varphi\right\}},
\end{aligned}
$$

where $X=T \exp \left(c_{0} Z\right), \Delta \varphi=\chi_{20}-\chi_{10}$.

In the $D(Z)=D_{0}=1, c_{0}=0$ limit, this solution is reduced to the well-known breather solution, which was found by Satsuma and Yajima (Satsuma \& Yajima, 1974) and was called as the Satsuma-Yajima breather:

$$
U_{2}(Z, T)=4 \frac{\cosh 3 T+3 \cosh T \exp (4 i Z)}{\cosh 4 T+4 \cosh 2 T+3 \cos 4 Z} \exp \left(\frac{i Z}{2}\right) .
$$

At $Z=0$ it takes the simple form $U(Z, T)=2 \operatorname{sech}(T)$. An interesting property of this solution is that its form oscillates with the so-called soliton period $T_{\text {sol }}=\pi / 2$.

In more general case of the varying dispersion, $D(Z)=D_{0} \exp \left(-c_{0} Z\right)$, shown in Fig.3 $\left(c_{0}=\right.$ $\left.0.25, \eta_{10}=0.25, \eta_{20}=0.75\right)$, the soliton period, according to Eq.(58), depends on time.

The Satsuma and Yajima breather solution can be obtained from the general solution if and only if the soliton phases are chosen properly, precisely when $\Delta \varphi=\pi$. The intensity profiles of the wave build up a complex landscape of peaks and valleys and reach their peaks at the points of the maximum. Decreasing group velocity dispersion (or increasing nonlinearity) stimulates the Satsuma-Yajima breather to accelerate its period of "breathing" and to increase its peak amplitudes of "breathing", that is why we call this effect as "agitated breather" in nonautonomous system. 

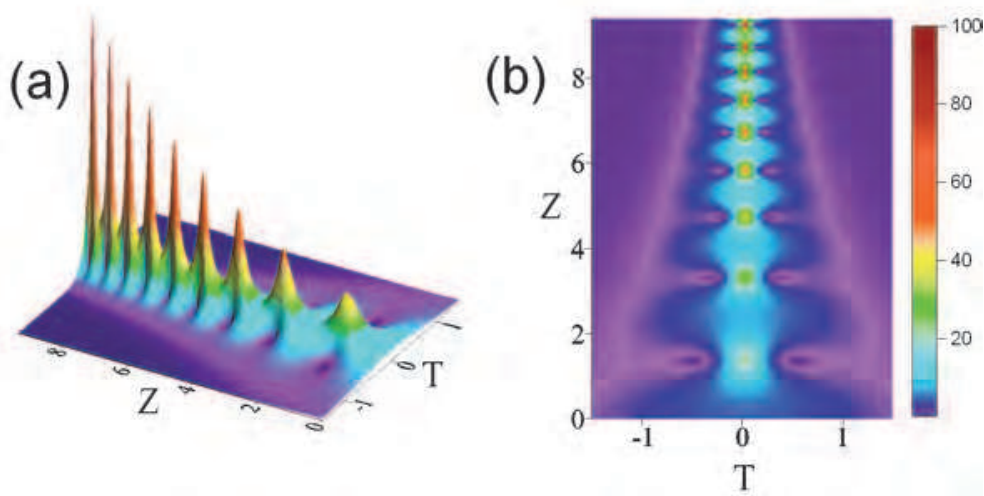

Fig. 3. Nonautonomous "agitated" breather (58) calculated within the framework of the model (46) after choosing the soliton management parameters $c_{0}=0.25, \eta_{10}=0.5, \eta_{20}=1.5$. (a) the temporal behavior; (b) the corresponding contour map.

\section{Rogue waves, "quantized" modulation instability, and dynamics of nonautonomous Peregrine solitons under "hyperbolic hurricane wind"}

Recently, a method of producing optical rogue waves, which are a physical counterpart to the rogue (monster) waves in oceans, have been developed (Solli et al., 2007). Optical rogue waves have been formed in the so-called soliton supercontinuum generation, a nonlinear optical process in which broadband "colored" solitons are generated from a narrowband optical background due to induced modulation instability and soliton fission effects (Dudley, 2009; Dudley et al., 2006; 2008).

Ordinary, the study of rogue waves has been focused on hydrodynamic applications and experiments (Clamond et al., 2006; Kharif \& Pelinovsky, 2003). Nonlinear phenomena in optical fibers also support rogue waves that are considered as soliton supercontinuum noise. It should be noticed that because optical rogue waves are closely related to oceanic rogue waves, the study of their properties opens novel possibilities to predict the dynamics of oceanic rogue waves. By using the mathematical equivalence between the propagation of nonlinear waves on water and the evolution of intense light pulses in optical fibers, an international research team (Kibler et al., 2010) recently reported the first observation of the so-called Peregrine soliton (Peregrine, 1983). Similar to giant nonlinear water waves, the Peregrine soliton solutions of the NLSE experience extremely rapid growth followed by just as rapid decay (Peregrine, 1983). Now, the Peregrine soliton is considered as a prototype of the famous ocean monster (rogue) waves responsible for many maritime catastrophes.

In this Section, the main attention will be focused on the possibilities of generation and amplification of nonautonomous Peregrine solitons. This study is an especially important for understanding how high intensity rogue waves may form in the very noisy and imperfect environment of the open ocean.

First of all, let us summarize the main features of the phenomenon known as the induced modulation instability. In 1984, Akira Hasegawa discovered that modulation instability of continuous (cw) wave optical signal in a glass fiber combined with an externally applied amplitude modulation can be utilized to produce a train of optical solitons (Hasegawa, 

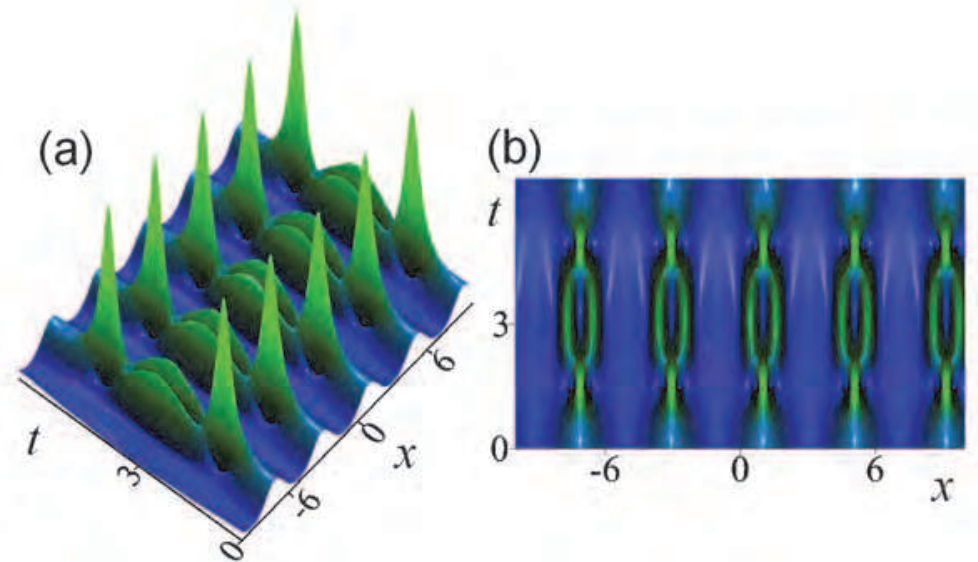

Fig. 4. Illustrative example of the temporal-spatial dynamics of the induced modulation instability and the Fermi-Pasta-Ulam recurrence effect calculated in the framework of the canonical NLSE model : (a) the intensity distribution; (b) the corresponding contour map.

1984). In the sense that the external modulation induces the modulation instability, Hasegawa called the total process as the induced modulation instability. To demonstrate the induced modulation instability (IMI), following Hasegawa, we solved the NLSE numerically with different depths and wavelength of modulation of cw wave. The main features of the induced modulation instability are presented in Fig.4. In Figure 4, following Hasegawa (Hasegawa, 1984), we present the total scenario of IMI and the restoration of the initial signal due to the Fermi-Pasta-Ulama recurrence effect. In our computer experiments, we have found novel and interesting feature of the IMI. Varying the depth of modulation and the level of continuous wave, we have discovered the effect which we called a "quantized" IMI. Figure 5 shows typical results of the computation. As can be clearly seen, the high-intensity IMI peaks are formed and split periodically into two, three, four, and more high-intensity peaks. In Fig.5 we present this splitting ("quantization") effect of the initially sinus like modulated cw signal into two and five high-intensity and "long-lived" components.

The Peregrine soliton can be considered as the utmost stage of the induced modulation instability, and its computer simulation is presented in Fig.6 When we compare the high-energy peaks of the IMI generated upon a distorted background (see Figs.4, 5) with exact form of the Peregrine soliton shown in Fig.7(a) we can understand, how such extreme wave structures may appear as they emerge suddenly on an irregular surface such as the open ocean.

There are two basic questions to be answered. What happens if arbitrary modulated cw wave is subjected to some form of external force? Such situations could include effects of wind, propagation of waves in nonuniform media with time dependent density gradients and slowly varying depth, nonlinearity and dispersion. For example, in Fig.7(b), we show the possibility of amplification of the Peregrine soliton when effects of wind are simulated by additional gain term in the canonical NLSE. The general questions naturally arise: To what extent the Peregrine soliton can be amplified under effects of wind, density gradients and 


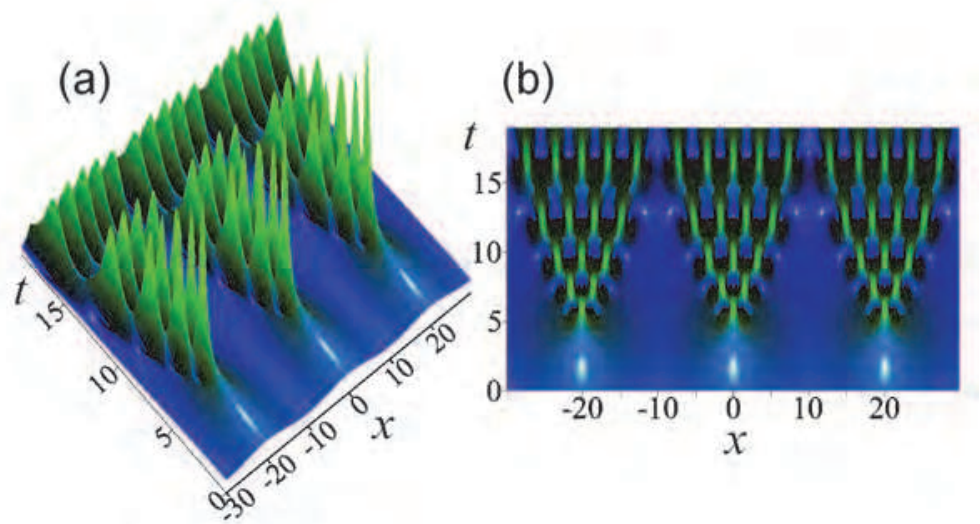

Fig. 5. Illustrative example of the "quantized" induced modulation instability: (a) the temporal-spatial behavior; (b) the corresponding contour map.

slowly varying depth, nonlinearity and dispersion? To answer these questions, let us consider the dynamics of the Peregrine soliton in the framework of the nonautonomous NLSE model. In the previous chapters, the auto -Bäcklund transformation has been used to find soliton solutions of the nonautonomous NLSE model. Now, we consider another remarkable method to study nonautonomous solitons. The following transformation

$$
q(x, t)=A(t) u(X, T) \exp [i \phi(X, T)]
$$

has been used by Serkin and Hasegawa in (Serkin \& Hasegawa, 2000a;b; 2002) to reduce the nonautonomous NLSE with varying dispersion, nonlinearity and gain or loss to the "ideal" NLSE

$$
i \frac{\partial u}{\partial T}+\frac{\sigma}{2} \frac{\partial^{2} u}{\partial X^{2}}+|u|^{2} u=0,
$$

where the following notations may be introduced

$$
\begin{gathered}
A(t)=\sqrt{P(t)} ; \quad X=P(t) x ; \quad T(t)=\int_{0}^{t} D(\tau) P^{2}(\tau) d \tau ; \\
\phi(X, T)=\frac{1}{2} \frac{W(R, D)}{R^{3}} X^{2}-\varphi(X, T),
\end{gathered}
$$

where $\varphi(X, T)$ is the phase of the canonical soliton.

It is easy to see that by using Eq.(59-61), the one-soliton solution may be written in the following form

$$
q_{1}^{+}(x, t \mid \sigma=+1)=2 \widetilde{\eta_{0}} A(t) \operatorname{sech}\left[2 \widetilde{\eta_{0}} X+4 \widetilde{\eta_{0}} \widetilde{\kappa_{0}} T(t)\right]
$$


(a)

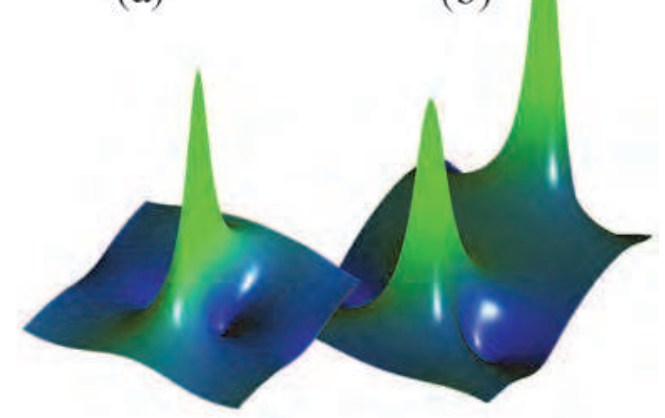

Fig. 6. Illustrative examples of the Peregrine soliton dynamics: (a) - classical Peregrine soliton calculated in the framework of the canonical NLSE model; (b) its behavior under linear amplification associated with continuous wind.

$$
\begin{aligned}
& \times \exp \left\{i\left[\frac{1}{2} \frac{W(R, D)}{R^{3}} X^{2}-2 \widetilde{\kappa_{0}} X-2\left({\widetilde{\kappa_{0}}}^{2}-{\widetilde{\eta_{0}}}^{2}\right) T(t)\right]\right\}, \\
\widetilde{\eta_{0}}= & \frac{D_{0}}{R_{0}} \eta_{0} ; \widetilde{\kappa_{0}}=\frac{D_{0}}{R_{0}} \kappa_{0} ; P(t)=R(t) / D(t) .
\end{aligned}
$$

The transformation (59) can be applied to obtain all solutions of the nonautonomous NLSE (30) and, in particular, the nonautonomous rational solutions known as the Peregrine solitons. Thus, the Peregrine soliton (Peregrine, 1983) can be discovered for the nonautonomous NLSE model as well

$$
q_{P}(x, t)=A(t) r(X, T) \exp [i \phi(T)]
$$

where

$$
\begin{aligned}
r(X, T) & =1-\frac{4(1+2 i T)}{1+4 T^{2}+4 X^{2}}, \\
\phi(X, T) & =\frac{1}{2} \frac{W(R, D)}{R^{3}} X^{2}+T(t)
\end{aligned}
$$

Figure 7 shows spatiotemporal behavior of the nonautonomous Peregrine soliton. The nonautonomous Peregrine soliton (63-65) shown in Fig.7(b) has been calculated in the framework of the nonautonomous NLSE model (28) after choosing the parameters $\lambda_{0}=\Omega=$ $0, D_{2}=R_{2}=1$ and the gain coefficient $\Gamma(t)=\Gamma_{0} /\left(1-\Gamma_{0} t\right)$. Somewhat surprisingly, however, this figure indicates a sharp compression and strong amplification of the nonautonomous Peregrine soliton under the action of hyperbolic gain which, in particular, in the open ocean can be associated with "hyperbolic hurricane wind".

It should be stressed that since the nonautonomous NLSE model is applied in many other physical systems such as plasmas and Bose-Einstein condensates (BEC), the results obtained in this Section can stimulate new research directions in many novel fields (see, for example, (Bludov et al., 2009; Yan, 2010)). 


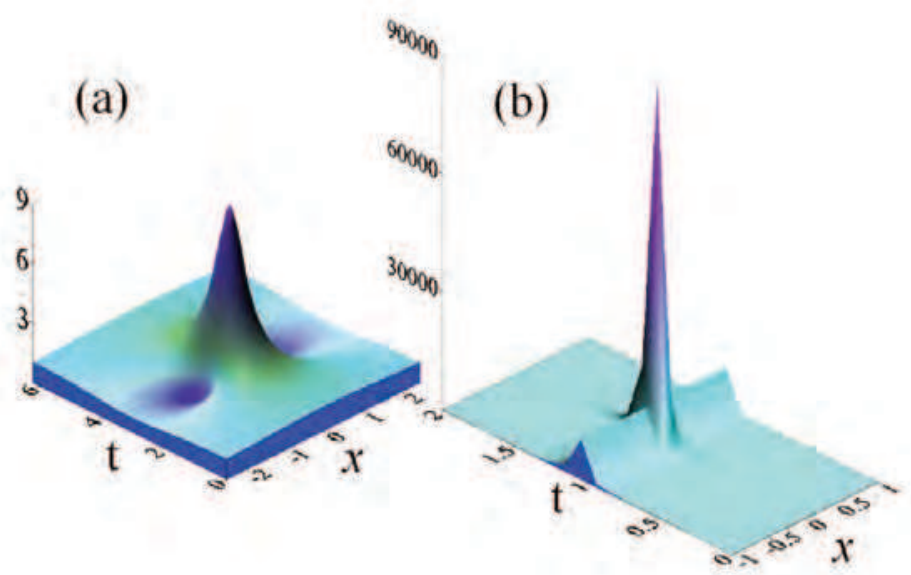

Fig. 7. (a) Autonomous and (b) nonautonomous Peregrine solitons calculated within the framework of the model (63-65) after choosing the soliton management parameters $\Gamma_{0}=0.33$.

\section{Nonautonomous $\mathrm{KdV}$ solitons}

Notice, that the nonlinear evolution equations that arise in the approach of variable spectral parameter contain, as a rule, an explicit dependence on the coordinates. Our general approach makes it possible to construct not only the well-known equations, but also a number of new integrable equations (NLSE, KdV, modified KdV, Hirota and Satsuma and so on) by extending the Zakharov-Shabat (ZS) and AKNS formalism. In particular, Eqs. $(9,10)$ under the conditions (11) with $a_{2}=0, a_{3}=-4 i D_{3}$ and $R=1$ become

$$
\begin{aligned}
Q_{T}= & -D_{3} Q_{S S S} S_{x}^{3}-6 i D_{3} Q_{S S} \varphi_{S} S_{x}^{3}+3 i D_{3} \sigma F^{2 \gamma} Q^{2} \varphi_{S} S_{x}+6 D_{3} \sigma F^{2 \gamma} Q Q_{S} S_{x} \\
& +Q_{S}\left(-S_{t}+\lambda_{1} S-V_{1} S_{x}-6 i D_{3} \varphi_{S S} S_{x}^{3}+\frac{3}{4} D_{3} \varphi_{S}^{2} S_{x}^{3}\right) \\
& -i Q\left[2 \lambda_{0} S / S_{x}-2 \gamma+\frac{1}{2}\left(\varphi_{T}+\varphi_{S} S_{t}\right)-\frac{1}{2} \lambda_{1} S \varphi_{S}+\frac{1}{2} V \varphi_{S} S_{x}\right] \\
& +Q\left(\lambda_{1}-\gamma \frac{F_{T}}{F}+\frac{3}{4} D_{3} \varphi_{S} \varphi_{S S} S_{x}^{3}\right)-i Q\left(-\frac{1}{8} D_{3} \varphi_{S}^{3} S_{x}^{3}+\frac{1}{2} D_{3} \varphi_{S S S} S_{x}^{3}\right),
\end{aligned}
$$

Eq.(66) can be rewritten in the independent variables $(x, t)$

$$
\begin{aligned}
Q_{t}= & -D_{3} Q_{x x x}-6 i D_{3} Q_{x x} \varphi_{x}+3 i D_{3} \sigma F^{2 \gamma} Q^{2} \varphi_{x}+6 D_{3} \sigma F^{2 \gamma} Q Q_{x} \\
& +Q_{x}\left(\lambda_{1} S / S_{x}-V_{1}-6 i D_{3} \varphi_{x x}+\frac{3}{4} D_{3} \varphi_{x}^{2}\right) \\
& -i Q\left[2 \lambda_{0} S / S_{x}-2 \gamma+\frac{1}{2}\left(\varphi_{T}+\varphi_{S} S_{t}\right)-\frac{1}{2} \lambda_{1} S \varphi_{x} / S_{x}+\frac{1}{2} V \varphi_{x}\right] \\
& +Q\left(\lambda_{1}-\gamma \frac{F_{t}}{F}+\frac{3}{4} D_{3} \varphi_{x} \varphi_{x x}\right)-i Q\left(-\frac{1}{8} D_{3} \varphi_{x}^{3}+\frac{1}{2} D_{3} \varphi_{x x x}\right) .
\end{aligned}
$$




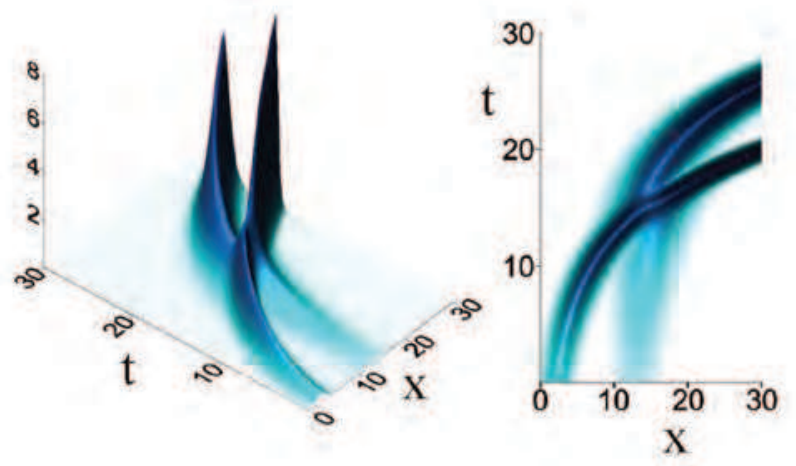

Fig. 8. Nonautonomous KdV solitons calculated within the framework of the model (71) after choosing the soliton management parameters $\alpha=0.15, \eta_{10}=0.40, \eta_{20}=0.75$. On the left hand side the temporal behavior is presented, while the corresponding contour map is presented on the right hand side.

Let us consider the simplest option to choose the real solution $Q(x, t)$, which leads to the only possibility of $\varphi=\lambda_{1}=0$. In this case, Eq.(67) is reduced to the $\mathrm{KdV}$ with variable coefficients

$$
Q_{t}-6 \sigma R_{3}(t) Q Q_{x}+D_{3}(t) Q_{x x x}+\frac{1}{2} \frac{W\left(D_{3}, R_{3}\right)}{D_{3} R_{3}}=0,
$$

where the notation $R_{3}(t)=F^{2 \gamma} D_{3}(t)$ has been introduced. It is easy to verify that Eq.(68) can be mapped into the standard $\mathrm{KdV}$ under the transformations

$$
Q(x, t)=\frac{D_{3}(T)}{R_{3}(T)} q(x, T),
$$

where $T=\int_{0}^{t} D_{3}(\tau) d \tau$ so that $q(x, T)$ is given by the canonical $\mathrm{KdV}$ :

$$
q_{t}-6 \sigma q q_{x}+q_{x x x}=0 .
$$

Applying the auto-Backlund transformation, we can write down the two-soliton solution of the nonautonomous $\mathrm{KdV}$

$$
Q_{2}(x, t)=-2 \sigma\left(\beta_{1}-\beta_{2}\right) \frac{D_{3}(T)}{R_{3}(T)} \frac{\mathfrak{N}_{1}}{\mathfrak{D}_{1}},
$$

where

$$
\begin{gathered}
\mathfrak{N}_{1}=\beta_{1}\left(\sinh \xi_{2}\right)^{2}+\beta_{2}\left(\cosh \xi_{1}\right)^{2}, \\
\mathfrak{D}_{1}=\left[\sqrt{2 \beta_{1}} \sinh \xi_{1} \sinh \xi_{2}-\sqrt{2 \beta_{2}} \cosh \xi_{1} \cosh \xi_{2}\right]^{2}, \\
\xi_{i}=\sqrt{\beta_{i} / 2}\left(x-2 \beta_{i} T\right), \beta_{i}=2 \eta_{0 i}^{2}, i=1,2 ;
\end{gathered}
$$

and $\eta_{02}>\eta_{01}$ are initial amplitudes of the solitons. 


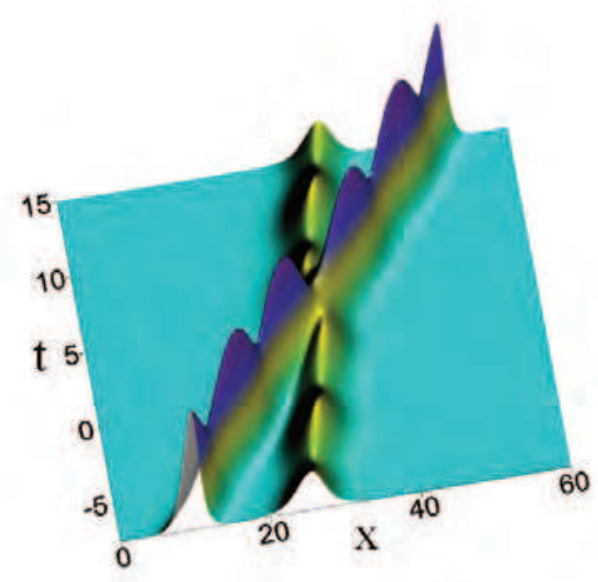

Fig. 9. Nonautonomous KdV solitons calculated within the framework of the model (72) after choosing the soliton management parameters $\alpha=2.0, \beta=-0.25, \eta_{10}=0.40, \eta_{20}=0.75$.

As two illustrative examples, in Fig.8, we present the behavior of nonautonomous KdV soliton in the framework of the model

$$
Q_{t}-6 \sigma Q Q_{x}+\exp (\alpha t) Q_{x x x}-\frac{1}{2} \alpha Q=0
$$

with lineal gain (or loss) accompanying by exponential variation of the dispersion coefficient; and in Fig.9 we show the dynamics of the $\mathrm{KdV}$ soliton in the nonautonomous system described by the model

$$
Q_{t}-6 \sigma Q Q_{x}+[1+\beta \cos (\alpha t)] /(1+\beta) Q_{x x x}+\frac{\alpha \beta \sin (\alpha t)}{2[1+\beta \cos (\alpha t)](1+\beta)} Q=0
$$

where $D_{3}(t)=[1+\beta \cos (\alpha t)] /(1+\beta), R_{3}(t)=1$.

It is important to compare our exactly integrable nonautonomous $\mathrm{KdV}$ model with the model proposed by Johnson to describe the KdV soliton dynamics under the influence of the depth variation (Johnson, 1997) and given by

$$
u_{X}-6 \sigma \mathcal{D}(X)^{-3 / 2} u u_{\xi}+\mathcal{D}(X)^{1 / 2} u_{\xi \xi \xi}+\frac{1}{2} \frac{\mathcal{D}_{X}}{\mathcal{D}} u=0 .
$$

We stress that after choosing the parameters $R_{3}(t)=\mathcal{D}(t)^{-3 / 2}$ and $D_{3}(t)=\mathcal{D}(t)^{1 / 2}$, the potential in Eq.(68) becomes $\frac{W\left(D_{3}, R_{3}\right)}{D_{3} R_{3}}=-2 \mathcal{D}^{\prime} / \mathcal{D}$, which is very nearly similar to the potential in Eq.(73) calculated by Johnson (Johnson, 1997).

\section{Conclusions}

The solution technique based on the generalized Lax pair operator method opens the possibility to study in details the nonlinear dynamics of solitons in nonautonomous nonlinear and dispersive physical systems. We have focused on the situation in which the generalized nonautonomous NLSE model was found to be exactly integrable from the point of view of 
the inverse scattering transform method. We have derived the laws of a soliton adaptation to the external potential. It is precisely this soliton adaptation mechanism which was of prime physical interest in our Chapter. We clarified some examples in order to gain a better understanding into this physical mechanism which can be considered as the interplay between nontrivial time-dependent parabolic soliton phase and external time-dependent potential. We stress that this nontrivial time-space dependent phase profile of nonautonomous soliton depends on the Wronskian of nonlinearity $R(t)$ and dispersion $D(t)$ and this profile does not exist for canonical NLSE soliton when $R(t)=D(t)=1$.

Several novel analytical solutions for water waves have been presented. In particular, we have found novel solutions for the generalized Peregrine solitons in inhomogeneous and nonautonomous systems, "quantized" modulation instability, and the exactly integrable model for the Peregrine solitons under "hyperbolic hurricane wind". It was shown that important mathematical analogies between optical rogue waves and the Peregrine solitons in water open the possibility to study optical rogue waves and water rogue waves in parallel and, due to the evident complexity of experiments with rogue waves in oceans, this method offers remarkable possibilities in studies nonlinear hydrodynamics problems by performing experiments in the nonlinear optical systems.

We would like to conclude by saying that the concept of adaptation is of primary importance in nature and nonautonomous solitons that interact elastically and generally move with varying amplitudes, speeds, and spectra adapted both to the external potentials and to the dispersion and nonlinearity changes can be fundamental objects of nonlinear science.

This investigation is a natural follow up of the works performed in collaboration with Professor Akira Hasegawa and the authors would like to thank him for this collaboration. We thank BUAP and CONACyT, Mexico for support.

\section{References}

Ablowitz, M. J., Kaup, D. J., Newell, A. C. \& Segur, H. (1973). Nonlinear-evolution equations of physical significance, Phys. Rev. Lett. 31(2): 125-127.

Agrawal, G. P. (2001). Nonlinear Fiber Optics, 3rd ed., Academic Press, San Diego.

Akhmediev, N. N. \& Ankiewicz, A. (1997). Solitons. Nonlinear pulses and beams, Charman and Hall, London.

Akhmediev, N. N. \& Ankiewicz, A. (2008). Dissipative Solitons: From Optics to Biology and Medicine, Springer-Verlag Berlin.

Atre, R., Panigrahi, P. K. \& Agarwal, G. S. (2006). Class of solitary wave solutions of the one-dimensional gross-pitaevskii equation, Phys. Rev. E 73(5): 056611.

Avelar, A. T., Bazeia, D. \& Cardoso, W. B. (2009). Solitons with cubic and quintic nonlinearities modulated in space and time, Phys. Rev. E 79(2): 025602.

Balakrishnan, R. (1985). Soliton propagation in nonuniform media, Phys. Rev. A 32(2): 1144-1149.

Belić, M., Petrović, N., Zhong, W.-P., Xie, R.-H. \& Chen, G. (2008). Analytical light bullet solutions to the generalized $(3+1)$-dimensional nonlinear schrödinger equation, Phys. Rev. Lett. 101(12): 123904.

Belyaeva, T., Serkin, V., Agüero, M., Hernandez-Tenorio, C. \& Kovachev, L. (2011). Hidden features of the soliton adaptation law to external potentials, Laser Physics 21: 258-263.

Bludov, Y. V., Konotop, V. V. \& Akhmediev, N. (2009). Matter rogue waves, Phys. Rev. A 80(3): 033610. 
Calogero, F. \& Degasperis, A. (1976). Coupled nonlinear evolution equations solvable via the inverse spectral transform, and solitons that come back: the boomeron, Lettere Al Nuovo Cimento 16: 425-433.

Calogero, F. \& Degasperis, A. (1982). Spectral transform and solitons: tools to solve and investigate nonlinear evolution equations, Elsevier Science Ltd.

Chen, H.-H. (1974). General derivation of bäcklund transformations from inverse scattering problems, Phys. Rev. Lett. 33(15): 925-928.

Chen, H. H. \& Liu, C. S. (1976). Solitons in nonuniform media, Phys. Rev. Lett. 37(11): 693-697.

Chen, H. H. \& Liu, C. S. (1978). Nonlinear wave and soliton propagation in media with arbitrary inhomogeneities, Phys. Fluids 21: 377-380.

Chen, S., Yang, Y. H., Yi, L., Lu, P. \& Guo, D. S. (2007). Phase fluctuations of linearly chirped solitons in a noisy optical fiber channel with varying dispersion, nonlinearity, and gain, Phys. Rev. E 75(3): 036617.

Christiansen, P. L., Sorensen, M. P. \& Scott, A. C. (2000). Nonlinear science at the dawn of the 21st century, Lecture Notes in Physics, Springer, Berlin.

Clamond, D., Francius, M., Grue, J. \& Kharif, C. (2006). Long time interaction of envelope solitons and freak wave formations, Eur. J. Mech. B/Fluids 25(5): 536-553.

Dianov, E. M., Mamyshev, P. V., Prokhorov, A. M. \& Serkin, V. N. (1989). Nonlinear Effects in Optical Fibers, Harwood Academic Publ., New York.

Dudley, J. M. \& Taylor, J. R. (2009). Ten years of nonlinear optics in photonic crystal fibre, Nature Photonics 3: 85-90.

Dudley, J. M., Genty, G. \& Coen, S. (2006). Supercontinuum generation in photonic crystal fiber, Rev. Mod. Phys. 78(4): 1135-1184.

Dudley, J. M., Genty, G. \& Eggleton, B. J. (2008). Harnessing and control of optical rogue waves insupercontinuum generation, Opt. Express 16(6): 3644-3651.

Gardner, C. S., Greene, J. M., Kruskal, M. D. \& Miura, R. M. (1967). Method for solving the korteweg-devries equation, Phys. Rev. Lett. 19(19): 1095-1097.

Gupta, M. R. \& Ray, J. (1981). Extension of inverse scattering method to nonlinear evolution equation in nonuniform medium, J. Math. Phys. 22(10): 2180-2183.

Hao, R. \& Zhou, G. (2008). Exact multi-soliton solutions in nonlinear optical systems, Optics Communications 281(17): 4474-4478.

Hasegawa, A. (1984). Generation of a train of soliton pulses by induced modulational instability in optical fibers, Opt. Lett. 9(7): 288-290.

Hasegawa, A. \& Kodama, Y. (1995). Solitons in Optical Communications, Oxford University Press.

Hasegawa, A. \& Matsumoto, M. (2003). Optical Solitons in Fibers, 3rd Edition, Springer-Verlag, Berlin.

He, X.-G., Zhao, D., Li, L. \& Luo, H. G. (2009). Engineering integrable nonautonomous nonlinear schrödinger equations, Phys. Rev. E 79(5): 056610.

Hernandez, T. C., Villargan, V. E., Serkin, V. N., Aguero, G. M., Belyaeva, T. L., Pena, M. R. \& Morales, L. L. (2005). Dynamics of solitons in the model of nonlinear schrödinger equation with an external harmonic potential: 1. bright solitons, Quantum Electronics 35(9): 778.

Hernandez-Tenorio, C., Belyaeva, T. L. \& Serkin, V. N. (2007). Parametric resonance for solitons in the nonlinear schrödinger equation model with time-dependent harmonic oscillator potential, Physica B: Condensed Matter 398(2): 460-463. 
Herrera, J. J. E. (1984). Envelope solitons in inhomogeneous media, J. Phys. A: Math. Gen. 17(1): 95-98.

Johnson, R. S. (1997). A modern introduction to the mathematical theory of water waves, Cambridge University Press, New York.

Kharif, C. \& Pelinovsky, E. (2003). Physical mechanisms of the rogue wave phenomenon, Eur. J. Mech. B. Fluid 22(6): 603-634.

Kharif, C., Pelinovsky, E. \& Slunyaev, A. (2009). Rogue Waves in the Ocean, Springer-Verlag, Berlin.

Kibler, B., Fatome, J., Finot, C., Millot, G., Dias, F., Genty, G., Akhmediev, N. \& Dudley, J. M. (2010). The peregrine soliton in nonlinear fibre optics, Nature Physics 6(10): 790-795.

Krumhansl, J. A. (1991). Unity in the science of physics, Physics Today 44(3): 33-38.

Lax, P. D. (1968). Integrals of nonlinear equations of evolution and solitary waves, Commun. on Pure and Applied Mathematics 21: 467-490.

Liu, W.-J., Tian, B. \& Zhang, H.-Q. (2008). Types of solutions of the variable-coefficient nonlinear schrödinger equation with symbolic computation, Phys. Rev. E 78(6): 066613.

Luo, H., Zhao, D. \& He, X. (2009). Exactly controllable transmission of nonautonomous optical solitons, Phys. Rev. A 79(6): 063802.

Nayfeh, A. H. \& Balachandran, B. (2004). Applied Nonlinear Dynamics, Wiley-VCH Verlag $\mathrm{GmbH} \& \mathrm{Co}$. KGaA, Weinheim.

Peregrine, D. H. (1983). Water waves, nonlinear schrödinger equations and their solutions, Austral. Math. Soc. Ser. B 25: 16-43.

Porsezian, K., Ganapathy, R., Hasegawa, A. \& Serkin, V. (2009). Nonautonomous soliton dispersion management, Quantum Electronics, IEEE Journal of 45(12): 1577-1583.

Porsezian, K., Hasegawa, A., Serkin, V., Belyaeva, T. \& Ganapathy, R. (2007). Dispersion and nonlinear management for femtosecond optical solitons, Phys. Lett. A 361(6): 504-508.

Satsuma, J. \& Yajima, N. (1974). Initial value problems of one-dimensional self-modulation of nonlinear waves in dispersive media, Prog. Theor. Phys. Supplement 55: 284-306.

Serkin, V. N. \& Belyaeva, T. L. (2001a). High-energy optical schrödinger solitons, JETP Letters 74: 573-577.

Serkin, V. N. \& Belyaeva, T. L. (2001b). The lax representation in the problem of soliton management, Quantum Electronics 31(11): 1007.

Serkin, V. N. \& Hasegawa, A. (2000a). Novel soliton solutions of the nonlinear schrödinger equation model, Phys. Rev. Lett. 85(21): 4502-4505.

Serkin, V. N. \& Hasegawa, A. (2000b). Soliton management in the nonlinear schrödinger equation model with varying dispersion, nonlinearity, and gain, JETP Letters 72: 89-92.

Serkin, V. N. \& Hasegawa, A. (2002). Exactly integrable nonlinear schrödinger equation models with varying dispersion, nonlinearity and gain: application for soliton dispersion, Selected Topics in Quantum Electronics, IEEE Journal of 8(3): 418 -431.

Serkin, V. N., Hasegawa, A. \& Belyaeva, T. L. (2004). Comment on "exact self-similar solutions of the generalized nonlinear schrödinger equation with distributed coefficients", Phys. Rev. Lett. 92(19): 199401.

Serkin, V. N., Hasegawa, A. \& Belyaeva, T. L. (2007). Nonautonomous solitons in external potentials, Phys. Rev. Lett. 98(7): 074102.

Serkin, V. N., Hasegawa, A. \& Belyaeva, T. L. (2010a). Nonautonomous matter-wave solitons near the feshbach resonance, Phys. Rev. A 81(2): 023610. 
Serkin, V. N., Hasegawa, A. \& Belyaeva, T. L. (2010b). Solitary waves in nonautonomous nonlinear and dispersive systems: nonautonomous solitons, Journal of Modern Optics 57(14-15): 1456-1472.

Serkin, V. N., Matsumoto, M. \& Belyaeva, T. L. (2001a). Bright and dark solitary nonlinear bloch waves in dispersion managed fiber systems and soliton lasers, Optics Communications 196(1-6): 159-171.

Serkin, V. N., Matsumoto, M. \& Belyaeva, T. L. (2001b). Nonlinear bloch waves, JETP Letters 73: 59-62.

Shin, H. J. (2008). Darboux invariants of integrable equations with variable spectral parameters, Journal of Physics A: Mathematical and Theoretical 41(28): 285201.

Solli, D. R., Ropers, C., Koonath, P. \& Jalali, B. (2007). Optical rogue waves, Nature 450: 1054-1057.

Tappert, F. D. \& Zabusky, N. J. (1971). Gradient-induced fission of solitons, Phys. Rev. Lett. 27(26): 1774-1776.

Taylor, J. R. (1992). Optical solitons - theory and experiment, Cambridge Univ. Press, Cambridge.

Tenorio, C. H., Villagran-Vargas, E., Serkin, V. N., Agüero-Granados, M., Belyaeva, T. L., Pena-Moreno, R. \& Morales-Lara, L. (2005). Dynamics of solitons in the model of nonlinear schrödinger equation with an external harmonic potential: 2. dark solitons, Quantum Electronics 35(10): 929.

Wang, J., Li, L. \& Jia, S. (2008). Nonlinear tunneling of optical similaritons in nonlinear waveguides, J. Opt. Soc. Am. B 25(8): 1254-1260.

Wu, L., Li, L. \& Zhang, J. F. (2008). Controllable generation and propagation of asymptotic parabolic optical waves in graded-index waveguide amplifiers, Phys. Rev. A 78(1): 013838.

Wu, L., Zhang, J.-F., Li, L., Finot, C. \& Porsezian, K. (2008). Similariton interactions in nonlinear graded-index waveguide amplifiers, Phys. Rev. A 78(5): 053807.

Yan, Z. (2010). Nonautonomous "rogons" in the inhomogeneous nonlinear schrödinger equation with variable coefficients, Physics Letters A 374(4): 672-679.

Zabusky, N. J. \& Kruskal, M. D. (1965). Interaction of "solitons" in a collisionless plasma and the recurrence of initial states, Phys. Rev. Lett. 15(6): 240-243.

Zakharov, V. E. (1980). The inverse scattering method, in R. Bullough \& P. J. Caudrey (eds), Solitons, Springer-Verlag, Berlin, pp. 243-285.

Zhang, J.-F., Wu, L. \& Li, L. (2008). Self-similar parabolic pulses in optical fiber amplifiers with gain dispersion and gain saturation, Phys. Rev. A 78(5): 055801.

Zhao, D., He, X.-G. \& Luo, H.-G. (2009). Transformation from the nonautonomous to standard nls equations, Eur. Phys. J. D 53: 213-216.

Zhao, D., Luo, H. G. \& Chai, H. Y. (2008). Integrability of the gross-pitaevskii equation with feshbach resonance management, Physics Letters A 372(35): 5644-5650. 


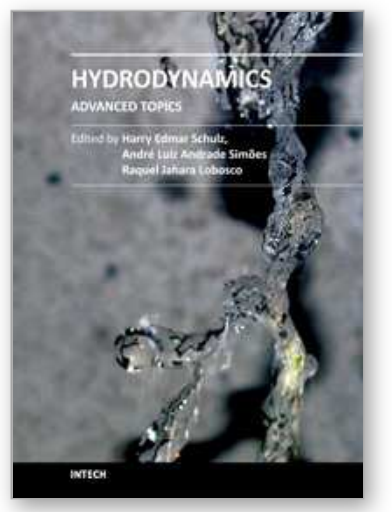

\author{
Hydrodynamics - Advanced Topics
}

Edited by Prof. Harry Schulz

ISBN 978-953-307-596-9

Hard cover, 442 pages

Publisher InTech

Published online 22, December, 2011

Published in print edition December, 2011

The phenomena related to the flow of fluids are generally complex, and difficult to quantify. New approaches considering points of view still not explored - may introduce useful tools in the study of Hydrodynamics and the related transport phenomena. The details of the flows and the properties of the fluids must be considered on a very small scale perspective. Consequently, new concepts and tools are generated to better describe the fluids and their properties. This volume presents conclusions about advanced topics of calculated and observed flows. It contains eighteen chapters, organized in five sections: 1) Mathematical Models in Fluid Mechanics, 2) Biological Applications and Biohydrodynamics, 3) Detailed Experimental Analyses of Fluids and Flows, 4) Radiation-, Electro-, Magnetohydrodynamics, and Magnetorheology, 5) Special Topics on Simulations and Experimental Data. These chapters present new points of view about methods and tools used in Hydrodynamics.

\title{
How to reference
}

In order to correctly reference this scholarly work, feel free to copy and paste the following:

T. L. Belyaeva and V. N. Serkin (2011). Nonautonomous Solitons: Applications from Nonlinear Optics to BEC and Hydrodynamics, Hydrodynamics - Advanced Topics, Prof. Harry Schulz (Ed.), ISBN: 978-953-307-596-9, InTech, Available from: http://www.intechopen.com/books/hydrodynamics-advanced-topics/nonautonomoussolitons-applications-from-nonlinear-optics-to-bec-and-hydrodynamics

\section{INTECH}

open science | open minds

\section{InTech Europe}

University Campus STeP Ri

Slavka Krautzeka 83/A

51000 Rijeka, Croatia

Phone: +385 (51) 770447

Fax: +385 (51) 686166

www.intechopen.com

\section{InTech China}

Unit 405, Office Block, Hotel Equatorial Shanghai

No.65, Yan An Road (West), Shanghai, 200040, China

中国上海市延安西路65号上海国际贵都大饭店办公楼 405 单元

Phone: +86-21-62489820

Fax: $+86-21-62489821$ 
(C) 2011 The Author(s). Licensee IntechOpen. This is an open access article distributed under the terms of the Creative Commons Attribution 3.0 License, which permits unrestricted use, distribution, and reproduction in any medium, provided the original work is properly cited. 